\title{
Transcriptome profiles revealed molecular mechanisms of alternating temperatures in breaking the epicotyl morphophysiological dormancy of Polygonatum sibiricum seeds
}

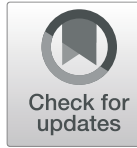

Dengqun Liao ${ }^{1 \dagger}$, Ruipeng $\mathrm{An}^{1,2,3 \dagger}$, Jianhe Wei ${ }^{1}$, Dongmei Wang ${ }^{2,3}$, Xianen $\mathrm{Li}^{1}$ and Jianjun $\mathrm{Qi}^{1^{*}}$ (D)

\begin{abstract}
Background: To adapt seasonal climate changes under natural environments, Polygonatum sibiricum seeds have a long period of epicotyl morphophysiological dormancy, which limits their wide-utilization in the large-scale plant progeny propagation. It has been proven that the controlled consecutive warm and cold temperature treatments can effectively break and shorten this seed dormancy status to promote its successful underdeveloped embryo growth, radicle emergence and shoot emergence. To uncover the molecular basis of seed dormancy release and seedling establishment, a SMRT full-length sequencing analysis and an Illumina sequencing-based comparison of $P$. sibiricum seed transcriptomes were combined to investigate transcriptional changes during warm and cold stratifications.
\end{abstract}

Results: A total of 87,251 unigenes, including 46,255 complete sequences, were obtained and 77,148 unigenes (88.42\%) were annotated. Gene expression analyses at four stratification stages identified a total of 27,059 DEGs in six pairwise comparisons and revealed that more differentially expressed genes were altered at the Corm stage than at the other stages, especially Str_S and Eme. The expression of 475 hormone metabolism genes and 510 hormone signaling genes was modulated during $P$. sibiricum seed dormancy release and seedling emergence. One thousand eighteen transcription factors and five hundred nineteen transcription regulators were detected differentially expressed during stratification and germination especially at Corm and Str_S stages. Of 1246 seed dormancy/germination known DEGs, 378, 790, and 199 DEGs were associated with P. sibiricum MD release (Corm vs Seed), epicotyl dormancy release (Str_S vs Corm), and the seedling establishment after the MPD release (Eme vs Str_S).

\footnotetext{
* Correspondence: jjqi@implad.ac.cn

${ }^{\dagger}$ Dengqun Liao and Ruipeng An contributed equally to this work.

${ }^{1}$ Institute of Medicinal Plant Development, Chinese Academy of Medical

Sciences \& Peking Union Medical College, Beijing 100193, China

Full list of author information is available at the end of the article
}

(c) The Author(s). 2021 Open Access This article is licensed under a Creative Commons Attribution 4.0 International License, which permits use, sharing, adaptation, distribution and reproduction in any medium or format, as long as you give appropriate credit to the original author(s) and the source, provide a link to the Creative Commons licence, and indicate if changes were made. The images or other third party material in this article are included in the article's Creative Commons licence, unless indicated otherwise in a credit line to the material. If material is not included in the article's Creative Commons licence and your intended use is not permitted by statutory regulation or exceeds the permitted use, you will need to obtain permission directly from the copyright holder. To view a copy of this licence, visit http://creativecommons.org/licenses/by/4.0/. The Creative Commons Public Domain Dedication waiver (http://creativecommons.org/publicdomain/zero/1.0/) applies to the data made available in this article, unless otherwise stated in a credit line to the data. 
Conclusions: A comparison with dormancy- and germination-related genes in Arabidopsis thaliana seeds revealed that genes related to multiple plant hormones, chromatin modifiers and remodelers, DNA methylation, mRNA degradation, endosperm weakening, and cell wall structures coordinately mediate $P$. sibiricum seed germination, epicotyl dormancy release, and seedling establishment. These results provided the first insights into molecular regulation of $P$. sibiricum seed epicotyl morphophysiological dormancy release and seedling emergence. They may form the foundation of future studies regarding gene interaction and the specific roles of individual tissues (endosperm, newly-formed corm) in P. sibiricum bulk seed dormancy.

Keywords: Polygonatum sibiricum red, Epicotyl morphophysiological dormancy, Temperature stratification, SMRT (single-molecule real-time) sequencing, Full-length transcriptome, RNA sequencing, Gene expression, Hormone, Transcription factor, Seed germination-related gene

\section{Background}

Polygonatum sibiricum Red (Huangjing in Chinese) is an edible perennial lily species with medicinal properties. The rhizome of this plant together with those of $P$. kingianum Col1. et Hemsl and P. cyrtonema Hua have been used as a traditional Chinese medicine for nourishing $Q i$ and Yin and for enhancing spleen, lung, and kidney functions for approximately 1600 years [1-4]. Modern pharmacological studies have proved that polygonati rhizoma can improve immunity as well as lower blood sugar and lipid levels. Additionally, its anti-viral and anti-tumorigenic properties have been confirmed. Thus, polygonati rhizoma may be useful for developing novel drugs and health products relevant for treating agerelated diseases, hypolipidemia, atherosclerosis, osteoporosis, liver diseases, diabetes mellitus, lung diseases, coughs, fatigue, and insomnia [3-8]. P. sibiricum is one of three Polygonatum species used as a source of polygonati rhizoma listed in the Pharmacopoeia of the People's Republic of China. As an edible and medicinal plant, the market demand for polygonati rhizoma has increased substantially, with more than 4000 tons produced annually. For many years, most of the polygonati rhizoma on the market was derived from wild resources, which has depleted the limited wild resources due to the long growth period to produce harvestable rhizomes (3-4 years for rhizome propagation and 5-6 years from seed propagation). To satisfy the increasing market demand and ensure the sustainable production and supply of polygonati rhizoma and to protect and preserve wild resources, Polygonatum plants are now widely cultivated in China. P. cyrtonema Hua is mainly cultivated in the Yangtze River basin and in the southern region, whereas P. kingianum Col1. et Hemsl and P. sibiricum Red are primarily cultivated in Yunnan province and northern China, respectively.

P. sibiricum can be propagated from its rhizomes or seeds. Because of the low efficiency of rhizome propagation and the considerable time needed for rhizome growth (3-4 years), seed propagation may be the superior option for the large-scale cultivation of P. sibiricum plants. However, P. sibiricum seeds at maturation have morphophysiological dormancy (MPD) and require a long dormancy period of about 15 months under natural conditions to complete the morphological and physiological dormancy-related processes before seedling emergence. It was found that seed structure, endogenous inhibitors, and underdeveloped embryo at maturation influenced $P$. sibiricum seed dormancy and germination [9]. Methods for effectively stimulating the germination of $P$. sibiricum dormant seeds, including soaking, exogenous hormone treatments, and temperature stratifications, were also evaluated. It was observed that $P$. sibiricum seeds exposed to $25^{\circ} \mathrm{C}$ could germinate in 30 60 days and form corm tissue, after which a lowtemperature $\left(4^{\circ} \mathrm{C}\right)$ treatment is needed to break the epicotyl dormancy in about 60 days before seedling emergence [10-12]. Similar to germination process of MPD seeds of Lilium dahuricum [13], Lilium polyphyllum [14], and Arisaema dracontium especially in cold regions $[15,16]$, P. sibiricum seeds underwent corm formation and plumule development, and required nutrients transported from endosperm reserves into the new corm tissue and a cold stratification prior to seedling establishment $[12,17]$. This type of seed dormancy and germination is quite different from the corresponding processes of many other MPD seeds such as Paris polyphylla [18], Panax quinquefolius [19], and Paeonia suffruticosa Andr [20]. For Paris polyphylla, Panax quinquefolius and Paeonia suffruticosa dormant seeds, their embryo differentiation into a visible radicle, plantule, hypocotyl or epicotyl, and/or cotyledons occurs inside the seed before germination. In contrast, the immature club-shaped embryo in $P$. sibiricum seeds elongates under suitable warm and moist conditions and pushes the radicle, hypocotyl, and plantule primordium out of the endosperm through the hilum; the visible plumule then differentiates and develops on the protuberant hypocotyl (defined as the "corm") and stops growing until a cold stratification is exerted to release epicotyl dormancy [12, 17] (Fig. 1). Currently, the molecular basis of $P$. sibiricum seed dormancy release and 


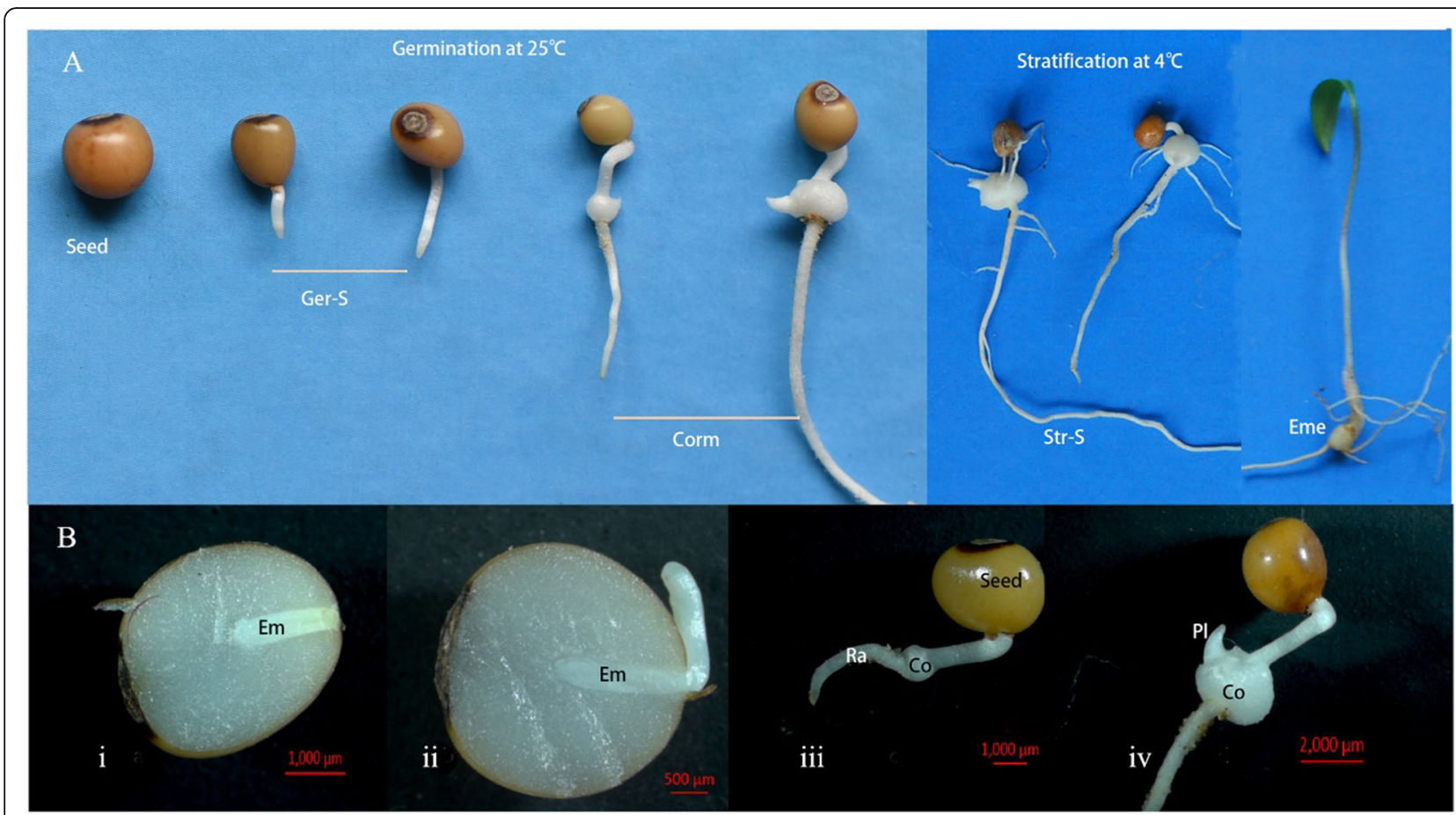

Fig. 1 Temperature stratification treatments promote P. sibiricum seed germination and seedling establishment (A) and embryo development (B) A five main overall stages of $P$. sibiricum seeds during stratification: dormant mature seed before a warm stratification (Seed); seed germination with the embryo extruding from a hilum (Ger-S) and cormlet formation (Corm) at $25^{\circ} \mathrm{C}$ stratification; the radicle and lateral roots form and the corm continues to grow at $4{ }^{\circ} \mathrm{C}$ stratification (Str_S); and seedling emergence with a leaf after transferring to $25^{\circ} \mathrm{C}$ (Eme). B, four major stages of embryo development during the germination at $25^{\circ} \mathrm{C}$ : i) undeveloped club-shaped embryo in a dormant seed at maturation; ii) immature embryo elongates and is extruded from the hilum, indicating the dormant $P$. sibiricum seed has germinated; iii) hypocotyl increases in size and a cormlet forms on the radicle; and iv) corm continues to grow and differentiate, which is accompanied by the emergence of a plumule. Em: embryo; Ra: radicle; Co: corm; Pl: plumule

germination, especially in terms of corm formation and epicotyl dormancy, remains unclear. Because of a lack of genome and transcriptome sequence information, in this study, we applied a single-molecule real-time sequencing (SMRT-seq) strategy to analyze a pooled total RNA sample derived from six stages to generate a complete and full-length $P$. sibiricum transcriptome during seed germination and seedling emergence. The transcript isoforms served as the reference sequences for the functional annotation of $P$. sibiricum genes and in the subsequent comparative transcriptome study. Additionally, we conducted an Illumina short-read sequencingbased comparison of the transcriptomic profiles in four key stages of $P$. sibiricum seeds during dormancy release. The potential genes related to the MPD release of $P$. sibiricum mature seeds following a warm stratification to develop the corm and the subsequent epicotyl dormancy release by a cold stratification were separately identified. These results provided the first insights into the molecular regulation of $P$. sibiricum seed MPD release, germination, and seedling emergence.

\section{Results}

\section{SMRT-seq analysis of $P$. sibiricum transcriptome during} seed dormancy, germination, and seedling emergence To obtain a sequenced $P$. sibiricum transcriptome during seed dormancy, germination, and seedling emergence, the PacBio RSII platform was used to perform a SMRTseq analysis of a pooled RNA sample from six different seed developmental stages [dormant mature seed, early germinating seed during a warm stratification (Ger-S), germinated seed with a corm during a warm stratification (Corm), early stage (about 4 weeks) of a cold stratification (Str), late stage (about 8 weeks) of a cold stratification (Str_S), and seedling emergence during a warm stratification (Eme)]. A total of 4,789,895 subreads (11.36 Gb) were generated, with an average length of $2373 \mathrm{bp}$ and an N50 of $3166 \mathrm{bp}$ (Table 1). A total of 292,791 circular consensus sequences (CCS) were obtained using the SMRTlink 5.1 software and further classified into 49,909 non-full length reads and 239,376 fulllength reads, of which 230,162 were full-length nonchimeric (Flnc) reads. An isoform-level clustering 
Table 1 Summary of the $P$. sibiricum transcriptome SMRT sequencing data

\begin{tabular}{ll}
\hline Total Subread base (Gb) & 11.36 \\
Subread number & $4,789,895$ \\
Average subread length (bp) & 2373 \\
N50 length (bp) & 3166 \\
Number of circular consensus sequence & 292,791 \\
Number of 5' primer reads & 267,809 \\
Number of 3' primer reads & 270,084 \\
Number of poly-A reads & 265,089 \\
Number of non-full-length reads & 49,909 \\
Number of full-length reads & 239,376 \\
Number of full-length non-chimeric reads & 230,162 \\
Average length of Flnc reads (bp) & 2920 \\
Number of consensus reads & 132,557 \\
Total nucleotides (bp) before and after correction & $404,700,846 / 40769,903$ \\
Mean length before and after correction (bp) & $3054 / 3076$ \\
Minimum length before and after correction (bp) & $161 / 162$ \\
Maximum length before and after correction (bp) & $14,162 / 14371$ \\
N50 length before and after correction (bp) & $3598 / 3633$ \\
N90 length before and after correction (bp) & $2059 / 2098$ \\
Number of unigenes & 87,251 \\
\hline
\end{tabular}

Note: $5^{\prime}$ primer reads refers to the reads with the $5^{\prime}$ primer; $3^{\prime}$ primer reads refers to the reads with the $3^{\prime}$ primer; poly-A reads refers to the reads with poly- $A$; fulllength non-chimeric (FInc) reads refers to non-chimeric reads with the $5^{\prime}$ primer, $3^{\prime}$ primer, and poly-A

analysis yielded 132,557 consensus reads, with an average length of $3076 \mathrm{bp}$, an N50 of $3633 \mathrm{bp}$, and an N90 of 2098 bp after correcting errors using RNA sequencing (RNA-seq) data. Redundant sequences among 132,557 consensus reads were eliminated using the CD-HIT software, ultimately resulting in 87,251 unigenes. Approximately $82.45 \%$ of all unigenes $(71,936)$ had only one transcript and $9.39 \%$ unigenes had two transcripts (Table $\mathrm{S} 1)$. The length distributions of the subreads, Flnc reads, and consensus sequences are presented in Fig. 2A-C. Additionally, 46,407 unigenes (53.19\% of the total) were longer than $3 \mathrm{kbp}$ (Fig. 2D). Using the ANGLE pipeline, 84,177 unigenes were predicted as protein-coding sequences, of which 46,255 were identified as full-length sequences (i.e., a complete coding sequence as well as $5^{\prime}$ and 3 ' untranslated regions). The length distribution of the predicted protein-coding sequences is provided in Figure S1.

\section{Functional annotation of $P$. sibiricum transcriptome}

A total of 87,251 full-length unigenes were functionally annotated based on BLAST searches of the NCBI nonredundant protein $(\mathrm{Nr})$, NCBI non-redundant nucleotide (Nt), Swiss-Prot, Protein family (Pfam), Clusters of Orthologous Groups (KOG/COG) of proteins, Gene Ontology (GO), and Kyoto Encyclopedia of Genes and Genomes (KEGG) databases. Using an E-cutoff value $\leq 1 \mathrm{e}-5$ and the top hit for the searches, a total of 77,148 unigenes $(88.42 \%)$ had significant sequence matches in at least one of the databases (Table S2). Among them, 25,521 unigenes $(29.25 \%)$ matched sequences in all seven databases. The number and percentage of annotated $P$. sibiricum unigenes for the seven databases were as follows: Nr: 74,921 (85.87\%), Nt: 54,583 (62.56\%), SwissProt: 61,678 (70.69\%), Pfam: 41,807 (47.92\%), KOG: 50,906 (58.34\%), GO: 41,807 (47.92\%), and KEGG: 73,525 (84.27\%). Among P. sibiricum unigenes annotated using the $\mathrm{Nr}$ database, 62,681 and 11,610 were assigned to 182 monocot species and 175 dicot species, respectively (Table S3). Among 402 Viridiplantae species with sequence matches, the three species with the most matches to $P$. sibiricum unigenes were Asparagus officinalis (39,017, 52.13\%), Elaeis guineensis (7561, 10.10\%), and Phoenix dactylifera (6226, 8.31\%), all of which are monocots. There are currently only 543 genes and 1732 protein sequences of Polygonatum species available in the NCBI databases. Our SMRT-seq analysis resulted in $544 P$. sibiricum unigenes assigned to the $\mathrm{Nr}$ sequences of the following eight Polygonatum species: P. biflorum (1), P. cyrtonema (175), P. involucratum (1), P. multiflorum (143), P. pubescens (7), P. roseum (64), P. sibiricum (73), and P. verticillatum (79) (Table S4). These 544 unigenes are $34.7-100 \%$ homologous to known sequences from Polygonatum species. 

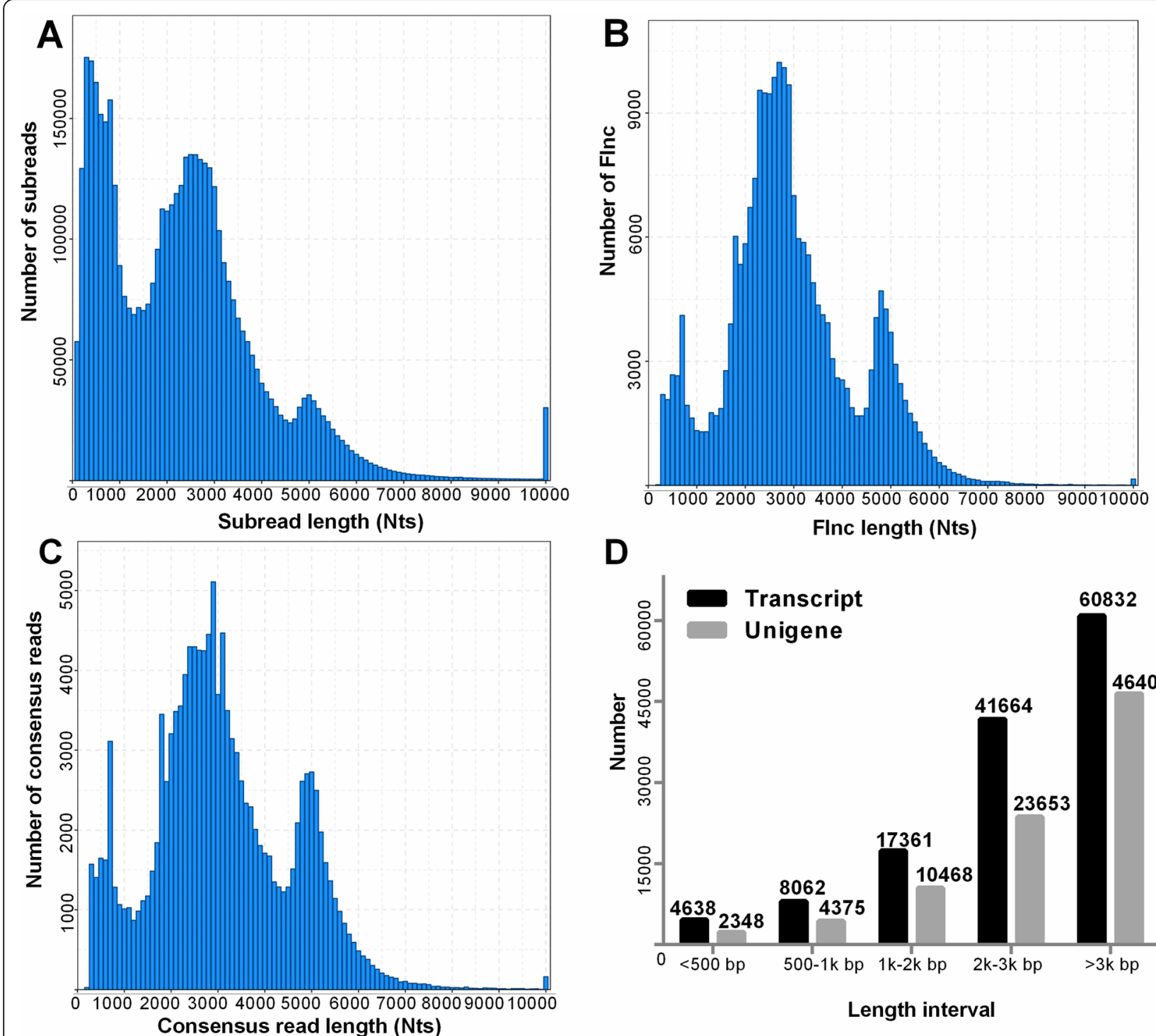

D

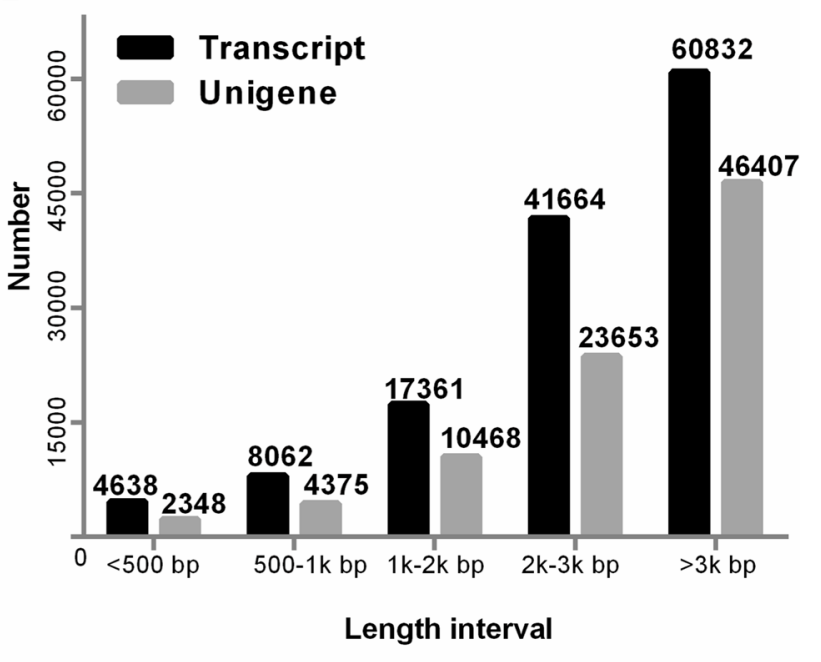

Fig. 2 Number and length distribution of PacBio SMRT sequencing data for P. sibiricum transcriptome. A Number and length distribution of subreads; B Number and length distribution of Flnc reads; C Number and length distribution of consensus reads (transcripts); $\mathbf{D}$ Distribution of the 132,557 transcripts and 82,571 unigenes at different length intervals

The KOG database was built with orthologous proteins encoded in the Arabidopsis thaliana (Arabidopsis) genome and the genomes of six other non-Viridiplantae species (http://www.ncbi.nlm.nih.gov/COG/). The BLASTx search of the KOG database matched 48,662 $P$. sibiricum unigenes with 7587 Arabidopsis orthologous proteins as well as 2244 P. sibiricum unigenes with 1279 orthologous proteins from non-Viridiplantae species. These 50,906 P. sibiricum unigenes were classified into 3209 orthologous protein clusters, and their distribution in 25 categories is presented in Figure S2. The largest KOG category was "general functional prediction only" $(13,408,15.37 \%)$, followed by "post-translational modification, protein turnover, chaperones" $(5295,6.07 \%)$ and "signal transduction mechanisms" (4766, 5.46\%). The GO functional annotation resulted in 41,807 unigenes classified into 54 subcategories (Table S5) of the three main functional categories (Figure S3). Specifically, 26,629 unigenes were assigned to 25 biological processes, with "metabolic process," "cellular process," and "single-organism process" revealed as the largest subcategories.

Among 73,525 P. sibiricum unigenes annotated using the KEGG database, 34,513 were assigned KO identifiers, of which 22,381 unigenes were further mapped to 341 KEGG pathways (third level) (Table S6). A total of 9323 unigenes were assigned to the metabolic pathways, including "carbohydrate metabolism" (3240), "energy 
metabolism" (1969), "lipid metabolism" (1506), and "amino acid metabolism" (1934). Additionally, 4225 unigenes were assigned to cellular processes, of which 2871 were associated with "transport and catabolism" and 1238 were related to "cell growth and death." Among the environmental information processing modules, 3862 unigenes were predicted to affect signal transduction pathways, including the "plant hormone signal transduction pathway" (699) and "MAPK signaling pathway-plant" (656), suggesting they may be useful for studying the regulatory effects of hormones on P. sibiricum seed germination.

\section{Differential expression of $P$. sibiricum genes during seed stratification}

To investigate the gene expression dynamics and patterns in P. sibiricum seeds during warm and cold stratifications and seedling establishment, four stages (Seed, Corm, Str_S, and Eme; Fig. 1) were analyzed by RNAseq using the Illumina PE150 platform, with three biological replicates for each stage. A total of 46-102 million clean reads were generated, of which 53.97-76.34\% were mapped to SMRT reference transcripts using the RSEM software (Table S7). Gene transcription level was assessed using FPKM value (i.e., expected number of fragments per kilobase of transcript sequence per million base pairs sequenced), which adjusted the transcript length and sequencing depth. The data indicated that $30.84-40.01 \%$ of the genes in 12 samples had FPKM values $<0.1$, whereas $32.71-39.86 \%$ of the genes had FPKM values >1(Figure S4A). Unigenes with FPKM values $>0.3$ were considered to be expressed. Accordingly, 62,984 genes were expressed in at least one of the 12 samples. The FPKM density distribution of the genes indicated that the overall gene expression of the Corm stage differed from that of the other three stages (Seed, Str_S, and Eme) (Figure S4B). The FPKM-based PCA confirmed that different $P$. sibiricum seed developmental stages were well separated, even though the three replicates for the Seed sample were more poorly clustered than the replicates for the other three stages (Figure S5A). A Pearson correlation analysis also revealed that the correlation between Seed2 and the Seed1 and Seed3 replicates $\left(R^{2}=0.68\right.$ and 0.66$)$ (Figure S5B) was lower than the correlation among replicates for the other three stages. Therefore, only Seed1 and Seed3 were used for analyzing differential gene expression. Pairwise comparisons revealed that $P$. sibiricum seeds at the Corm stage had substantially more differentially expressed genes (DEGs), especially compared with the seeds at the Str_S and Eme stages (Fig. 3A). Using an adjusted $p$ value $<$ 0.05 and $\mid \log _{2}$ (fold-change) $\mid \geq 1$ as the criteria, 7248, 17,619 , and 19,319 DEGs were respectively detected in comparisons between the Corm stage and the Seed, Str S, and Eme stages. Only 3183 and 1170 unigenes were differentially expressed between the Eme and Seed stages and between the Str_S and Seed stages, respectively. These results imply that imbibed $P$. sibiricum seeds with undeveloped embryos had physiological activities that were similar to those of $P$. sibiricum cold-stratified seeds and seedlings after the dormancy constraints in the Corm stage seeds were eliminated or alleviated by cold stratification. Of 27,059 DEGs, 46-5411 were differentially expressed only between two stages. Additionally, 8825 and 6428 DEGs were detected in two and three comparisons, respectively (Fig. 3B).

A hierarchical clustering analysis (Fig. 4A) classified 27,059 DEGs into eight main subclusters. Compared

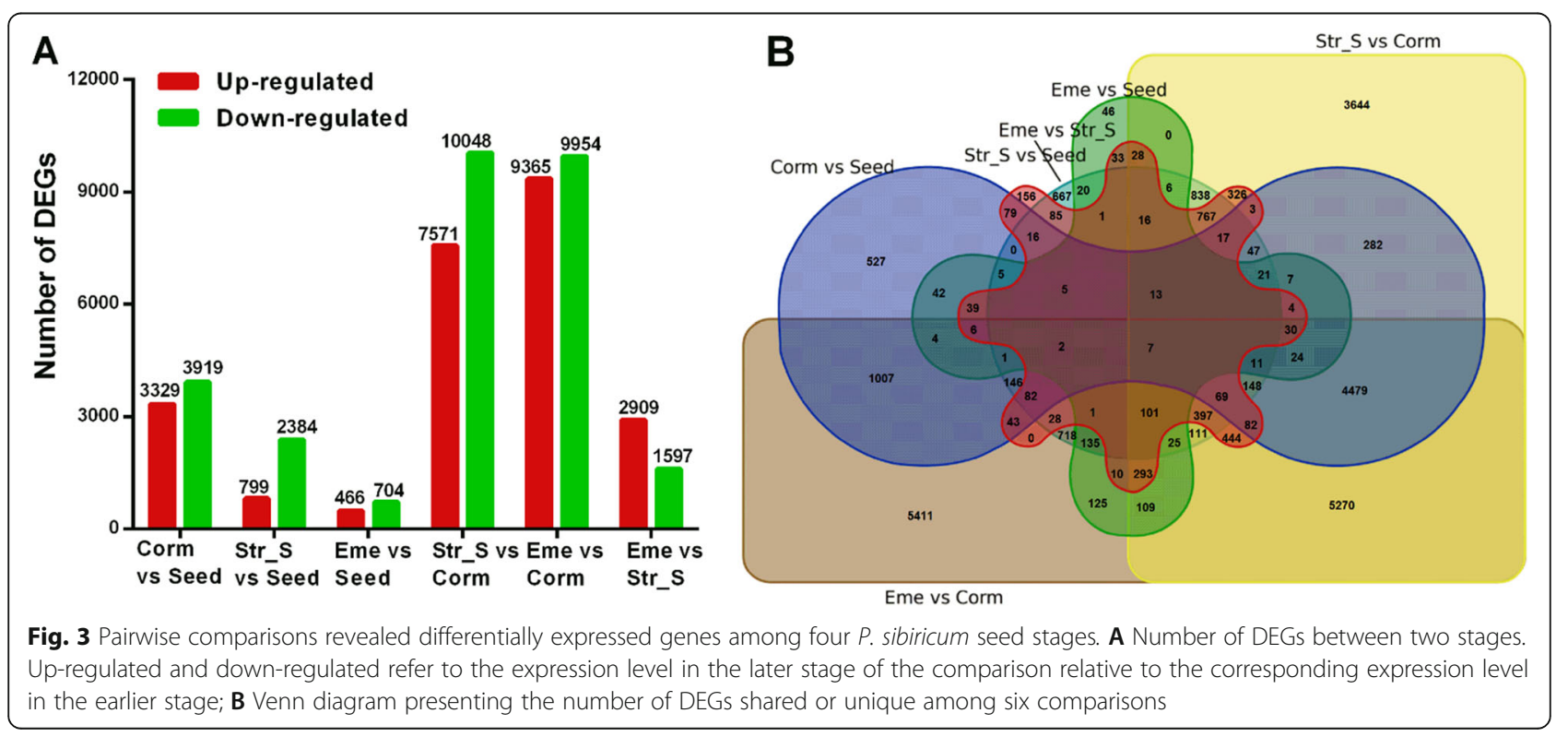




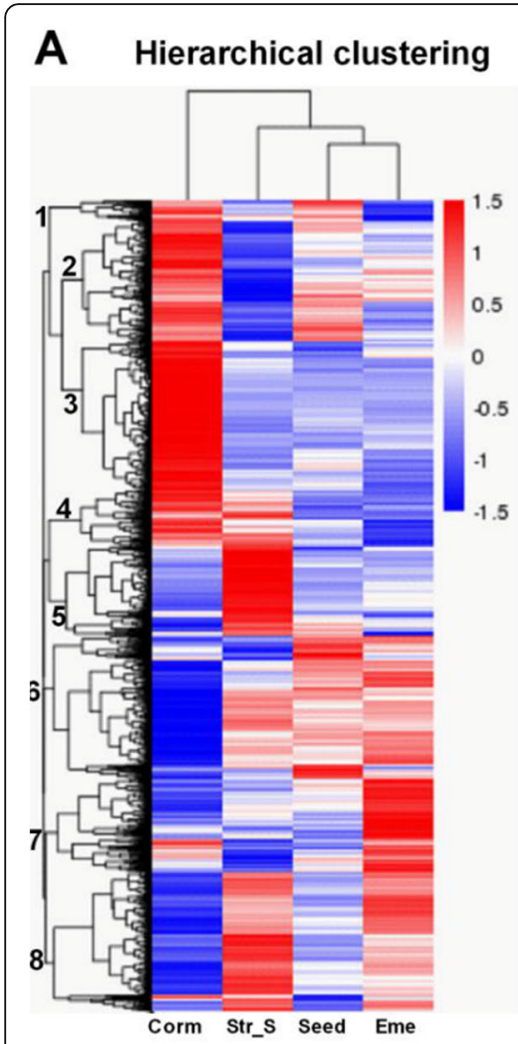

\section{B K-medoids clustering}
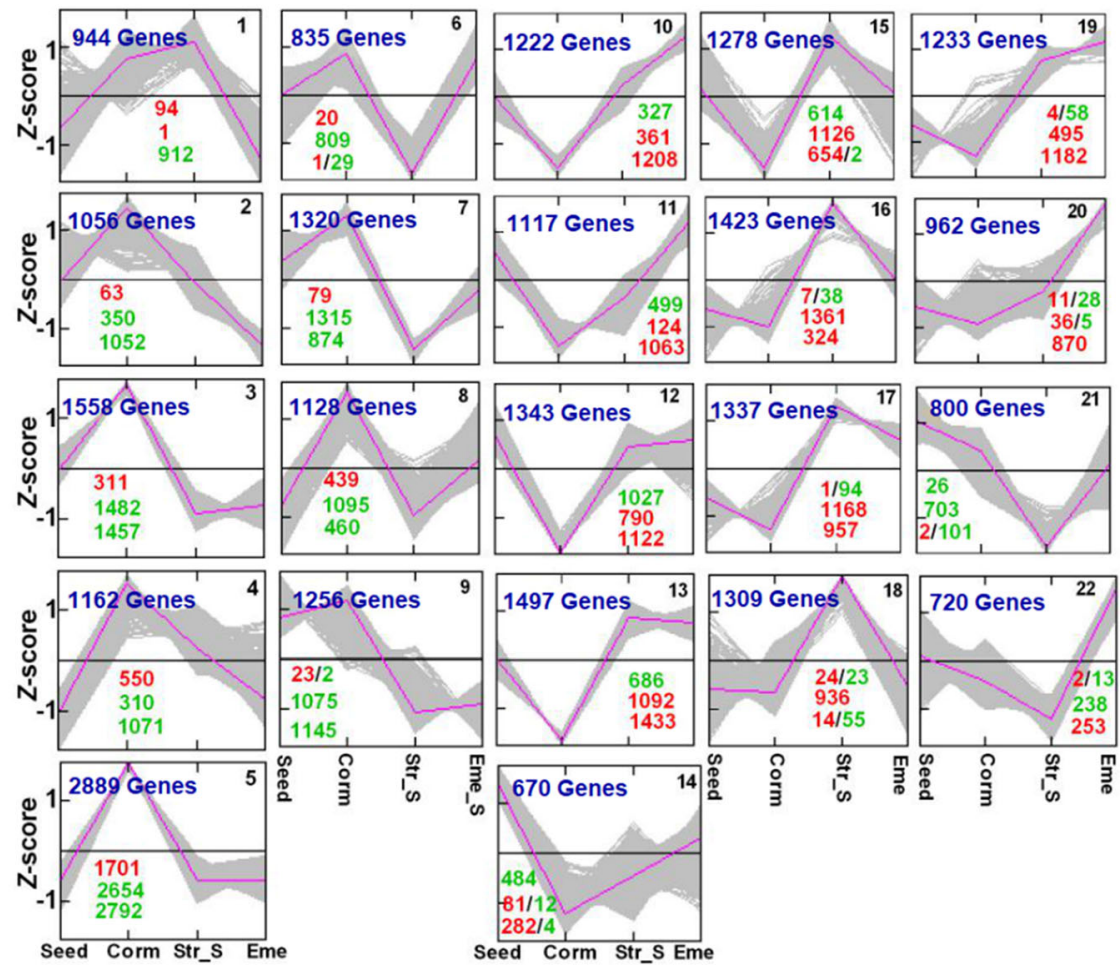

Fig. 4 Expression patterns of P. sibiricum DEGs during seed dormancy release and seedling establishment. A Hierarchical clustering of all DEGs. Red and blue represent increased and decreased transcript abundances, respectively. The eight main subclusters are indicated at the branching points (left side). B Results of the K-medoids clustering. The Z-scores of $\log _{2}$ (FPKM+1) were used for the K-medoids clustering with the MEV software (v. 4.9.0) and Pearson correlations. The subcluster number is provided in the upper right corner of each subgraph. The numbers in dark blue indicate the number of DEGs in each subcluster. The numbers of DEGs in the Corm vs Seed, Str_S vs Corm, and Eme vs Corm comparisons are summarized (top to bottom) under the expression curves for each subcluster. Red and green numbers represent the number of transcripts with increased and decreased abundances, respectively

with the expression levels in the Seed, Str_S, and Eme stages, most genes in Subclusters 1-4 were more highly expressed at the Corm stage, whereas most genes in Subclusters 5-8 were expressed at lower levels. A subsequent K-medoids analysis (Fig. 4B) classified 27,059 DEGs into 22 subclusters. The expression of the DEGs in Subclusters 1-9 increased mainly at the Corm stage during a warm stratification and then decreased at the end of cold stratification or during seedling emergence, whereas the expression of the DEGs in Subclusters 1022 generally had the opposite pattern. Specifically, Subclusters 1-8 included 3257 DEGs with up-regulated expression in the Corm vs Seed comparison, whereas Subclusters 10-15 comprised 3637 DEGs with downregulated expression in the Corm vs Seed comparison. Moreover, 7571 and 10,048 DEGs with up- and downregulated expression, respectively, were detected in the Str_S vs Corm comparison: 7543 of the 7571 DEGs were in Subclusters 10-19 (except Subcluster 14) and 9090 of the 10,048 DEGs were in Subclusters 2-8. Furthermore, 9763 DEGs with down-regulated expression in the Eme vs Corm comparison were in Subclusters 1-9 (except for Subcluster 6), whereas 9348 DEGs with up-regulated expression were in Subclusters 10-22 (except for Subclusters 18 and 21).

The KEGG pathway enrichment analyses of the $P$. sibiricum DEGs in the Corm vs Seed, Str_S vs Corm, Eme vs Corm, and Eme vs Str_S comparisons involved the top 20 pathways and $-\log _{10}$ (adjusted $p$ value) of "all DEGs" sets in each comparison (Fig. 5). The enriched pathways among the top 20 KEGG pathways differed greatly among the Str_S vs Corm, Eme vs Corm, and Eme vs Str_S comparisons. Only two KEGG pathways, "DNA replication" and "Spliceosome," were enriched for the "all DEGs" set in the Corm vs Seed comparison (Fig. 5A). Many enriched KEGG pathways were identified for the "all DEGs" set and for the "down-regulated" gene set in the Str_S vs Corm comparison (Fig. 5B) or for the "up-regulated" gene set in the Eme vs Str_S comparison (Fig. 5D). Thirteen KEGG pathways, including "starch and sucrose metabolism," were enriched for the "downregulated" gene set, whereas three KEGG pathways, 

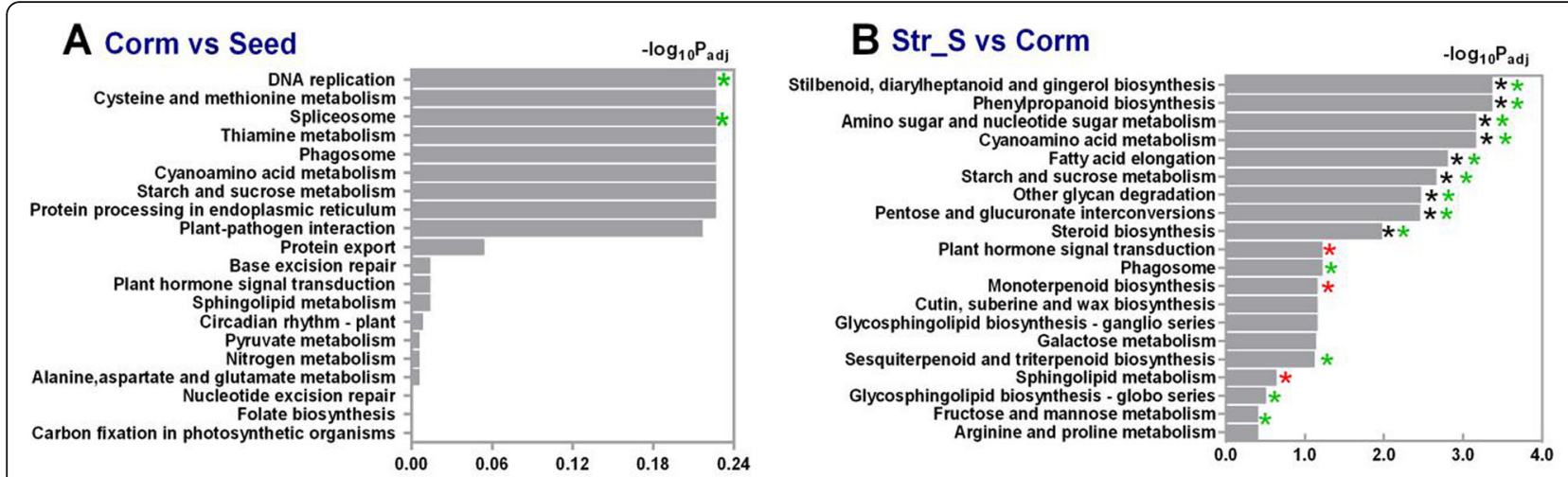

\section{Eme vs Corm}

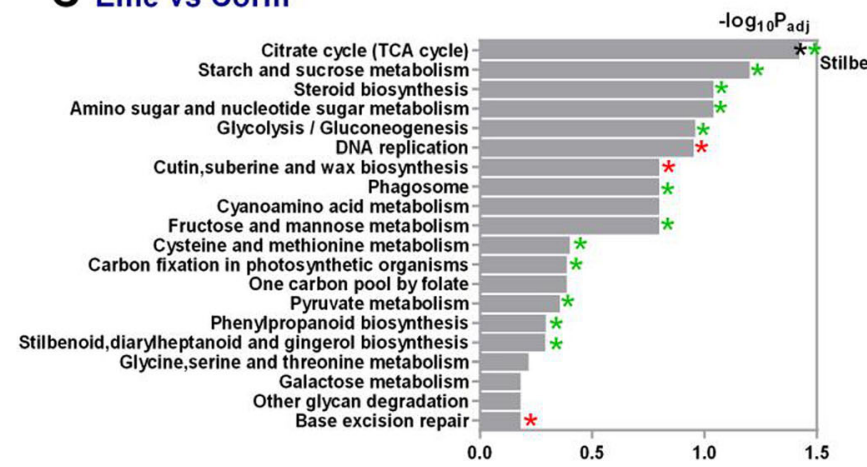

\section{Eme vs Str_S}

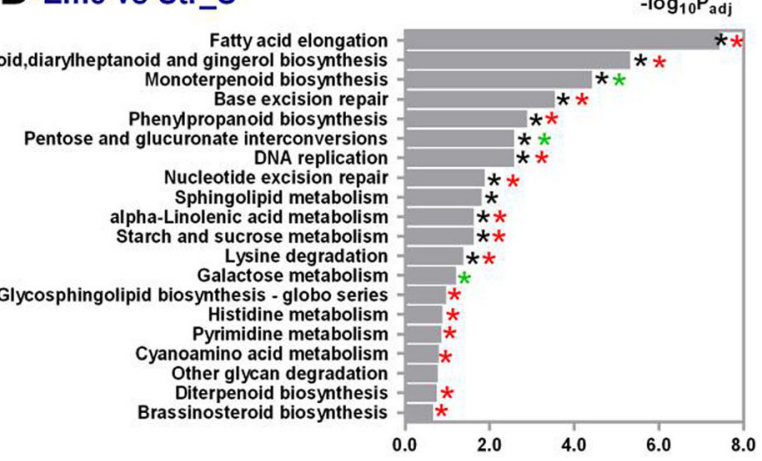

Fig. 5 KEGG pathway enrichment analyses of P. sibiricum DEGs between Seed and Corm (A), Corm and Str_S (B), Corm and Eme (C), and Eme and Str_S (D) stages. A KEGG enrichment map was constructed based on the top 20 pathways and - $\log _{10}$ (adjusted $p$ value) of the "all DEGs" sets in each comparison. * * and * (adjusted $p$ value $<0.05$ ) indicates the pathway was significantly enriched for the "all DEGs," "up-regulated," and "down-regulated" sets, respectively

including "plant signal hormone transduction," were enriched for the "up-regulated" gene set in the Str_S vs Corm comparison (Fig. 5B). Additionally, 15 KEGG pathways were significantly enriched for the "up-regulated" gene set, but only three enriched KEGG pathways were identified for the "down-regulated" gene set in the Eme vs Str_S comparison (Fig. 5D). The enriched KEGG pathways in the Eme vs Corm comparison, including "starch and sucrose metabolism" and "steroid biosynthesis," were mainly associated with the "down-regulated" gene set (Fig. 5C).

\section{Expression of hormone metabolism and signaling genes during $P$. sibiricum seed dormancy and germination}

A BLASTx search of the Arabidopsis protein database identified 1189 P. sibiricum unigenes possibly involved in the metabolism of ABA, GA, auxin, BR, and other hormones or the upstream pathways (Table S8). Additionally, 1285 putative hormone signaling genes were identified based on the KEGG annotations and published relevant information for Arabidopsis (Table S8). The expression levels of 475 hormone metabolism genes and 510 hormone signaling genes changed during $P$. sibiricum seed dormancy release and seedling emergence (Table S8, Figure S6). Figures 6 and 7 present the expression levels of the selected DEGs involved in the biosynthesis, degradation, and signaling of ABA (51), GA (30), CK (30), auxin (60), BR (39), JA (54), and ethylene (37) in four samples. The expression levels of many ABA signaling genes (13 of 34) were up-regulated in the Corm, Str_S, and Eme stages (Fig. 6A), reflecting the importance of $\mathrm{ABA}$ signaling for seed germination and dormancy release. The CYP707A1 gene, which is involved in ABA degradation, was more highly expressed in the Corm (c9540), Str_S (c60708), and Eme (c29776) stages than in the Seed stage. The expression of a GA3ox gene (c4067) involved in GA biosynthesis was upregulated in the Seed and Corm stages, especially compared with that in the Eme stage, whereas the expression of a GA2ox gene (c8451) involved in GA degradation was up-regulated in the Corm and Eme stages (Fig. 6B).

Fifteen ABA- and GA-related genes were selected for a quantitative real-time polymerase chain reaction (qRTPCR) analysis to verify the accuracy of the RNA-seqbased expression levels during the P. sibiricum seed dormancy release process (Figures S7 and S8). We analyzed 

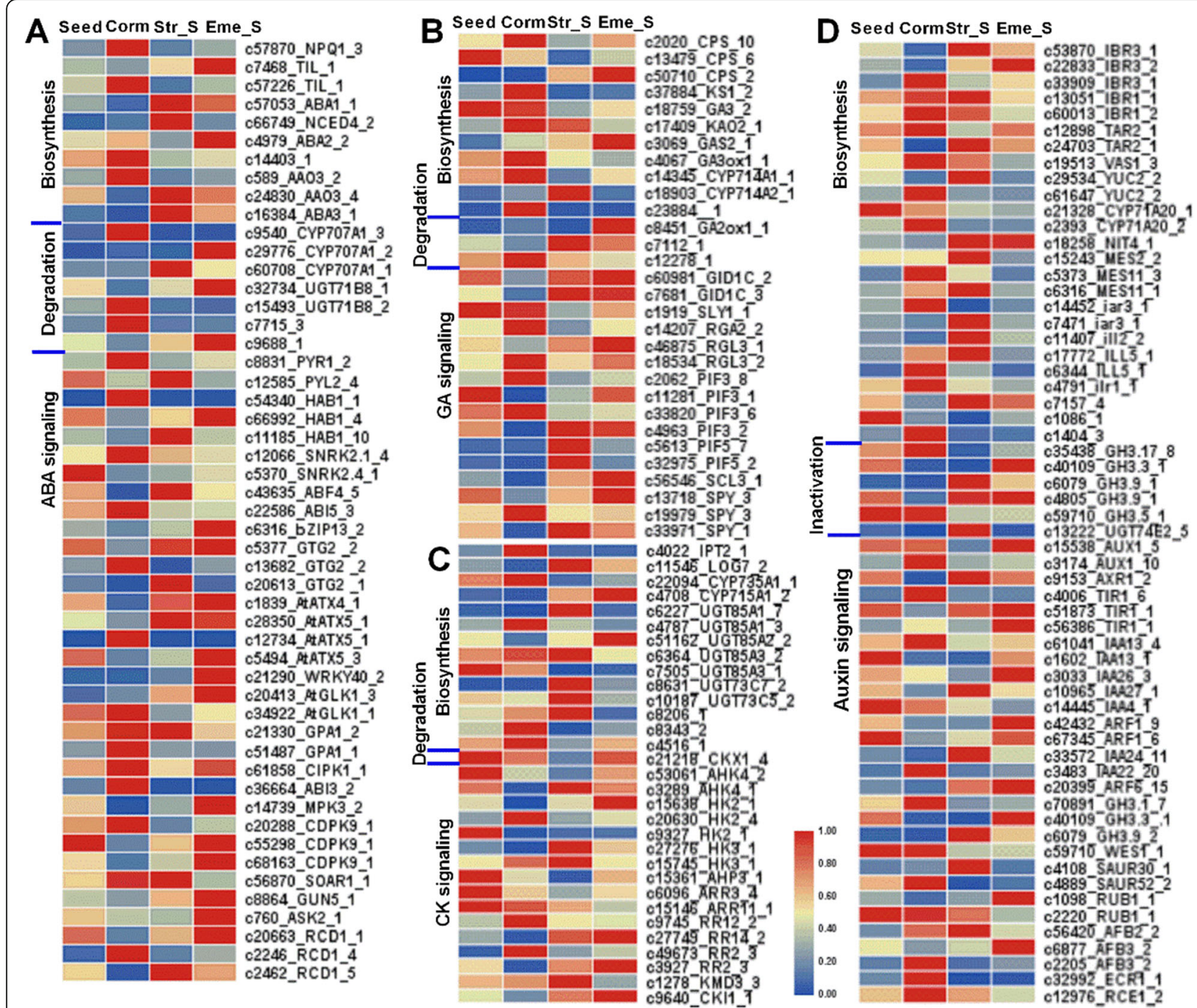

Fig. 6 Representative expression patterns of P. sibiricum DEGs involved in ABA (A), GA (B), CK (C), and auxin (D) metabolism and signal transduction pathways. To calculate the relative expression level of a gene, its FPKM value was divided by the maximum FPKM value for that gene over four sampling stages. Heatmaps of selected DEGs were constructed using TBools without gene or group clustering. The genes are listed based on their positions in the hormone metabolism and signal transduction pathways. Partial IDs and abbreviated gene names as well as the number of DEGs in the same enzyme or functional family with similar expression profiles are presented on the right. Specific details are provided in Table $\mathrm{S} 8$

four seed dormancy release stages, three involving a stratification at $25^{\circ} \mathrm{C}$ (Seed, Corm, and Eme) and one low-temperature stage (Str_S). The FPKM values indicated that two ABA biosynthesis-related genes, ZEP (zeaxanthin epoxidase) and $A A O 3$ (abscisic-aldehyde oxidase 3 ), and one ABA degradation-related gene $(C Y P 707 A)$ had consistent expression trends in the four samples. More specifically, ZEP and CYP707A were expressed at low levels in the Corm stage, in contrast to the relatively high expression level at the lowtemperature stage (Str_S). These results indicate that an exposure to low temperatures is important for breaking the P. sibiricum epicotyl dormancy, which is required to complete the seed epitcotyl dormancy release process. The ABA signaling genes, including $A B I 5$ [basic leucine zipper (bZIP) transcription factor (TF)], $A B F$ (ABA-responsive element-binding factor), PYL4 (polyketide cyclase/dehydrase and lipid transport superfamily protein), $P Y L 8$, and $P P 2 C$ (protein phosphatase $2 \mathrm{C}$ ), were also analyzed by qRT-PCR. Their RT-qPCR results were basically consistent with the RNA-seq data. Two GA synthesis genes [GA3ox and GAMT2 (gibberellic acid methyltransferase 2)], five GA signaling genes [GASA3 (gibberellin-regulated protein 3), GASA6, GASA14, GID1, and GID1-like (gibberellin receptor)], and CIGR2 (chitin-inducible gibberellin-responsive protein 2) also 

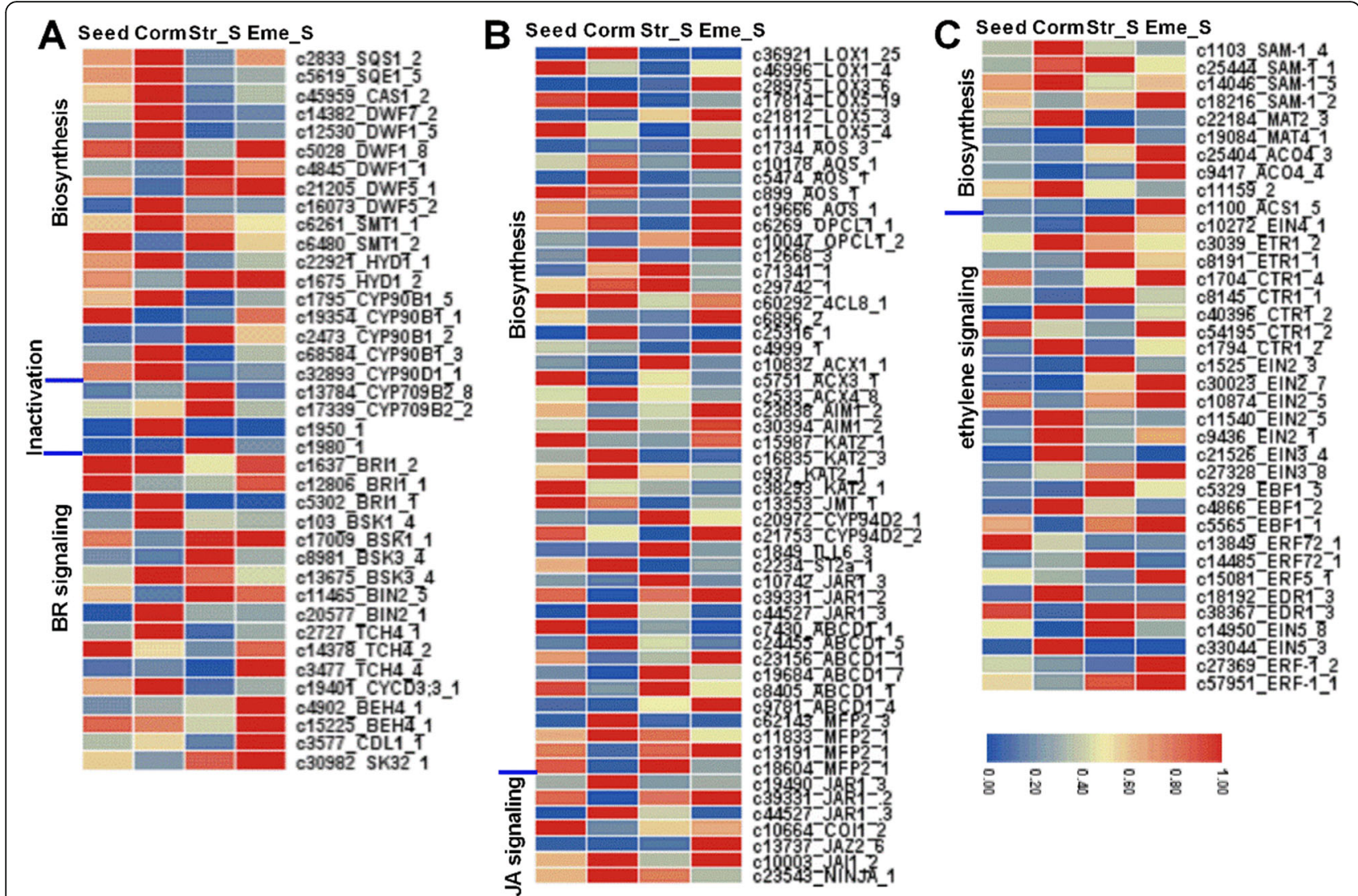

Fig. 7 Representative expression patterns of P. sibiricum DEGs involved in BR (A), JA (B), and ethylene (C) metabolism and signal transduction pathways. To calculate the expression level of a gene, its FPKM value was divided by the maximum FPKM value for that gene over four sampling stages. Heatmaps of selected DEGs were constructed using TBools without gene or group clustering. The genes are listed based on their positions in the hormone metabolism and signal transduction pathways. Partial IDs and abbreviated gene names as well as the number of DEGs in the same enzyme or functional family with similar expression profiles are presented on the right. Specific details are provided in Table S8

showed the consistent qRT-PCR and FPKM values (Figure S8). The GA3ox expression level was up-regulated at the Corm stage, whereas GAMT2 expression was upregulated at the Seed and Eme stages. The GASA3, GASA6, and GASA14 genes were differentially expressed. Moreover, GID1 and CIGR2 were expressed at low levels during the Seed and Corm stages, but were highly expressed at the Str_S and Eme stages.

\section{Expression of transcription factors during $P$. sibiricum seed dormancy and germination}

Transcription factors are critical for seed development and germination [21-23]. In this study, we annotated our transcriptome using the iTAK software and the associated database, ultimately identifying 2605 TFs from 67 TF families as well as 1552 transcriptional regulators (TRs) from 25 TR families (Tables S9, S10). Among them, 1018 TFs (57 TF families) and 519 TRs (21 TR families) were differentially expressed during $P$. sibiricum seed stratification and germination. A hierarchical clustering analysis performed using the FPKM values of the TF and TR DEGs produced 10 subclusters (Fig. 8 and Table S9). These 10 subclusters were classified into two main groups based on the relative expression levels in the Corm samples: Subcluster 1-6 (542 TFs/ 273 TRs) in Corm with decreased expression while Subcluster 7-10 (476 TFs/246 TRs) having higher expression. -Some Arabidopsis TF/TR genes were functionally known to play roles in seed dormancy and germination (Table S11). The possible roles of the differentially expressed TFs and TRs are listed in Table S11 and are described in more detail below.

\section{Analysis of $P$. sibiricum DEGs related to seed dormancy release based on known Arabidopsis genes}

To date, a total of 630 Arabidopsis genes have been functionally verified to be involved in seed dormancy and germination (Table S11). A BLASTx search identified that 3407 P. sibiricum unigenes, including 688 encoding TFs or TRs are homologs of 413 of these known Arabidopsis genes. An analysis of differential gene expression based on the FPKM values of four samples 


\section{A Hierarchical clustering B Statistics}

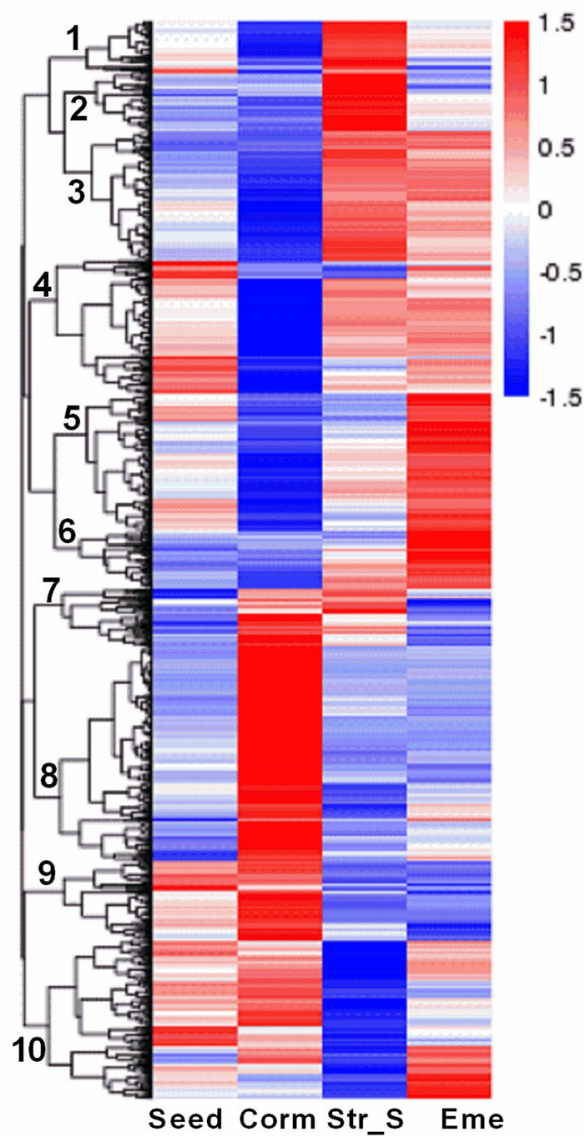

\begin{tabular}{|c|c|c|}
\hline $\begin{array}{l}\text { Cluster } \\
\text { name }\end{array}$ & $\begin{array}{l}\text { Number } \\
\text { of DEGs }\end{array}$ & $\begin{array}{l}\text { Number of } \\
\text { TF(TR) } \\
\text { families }\end{array}$ \\
\hline $\mathrm{C} 1$ & 72 & 32 \\
\hline $\mathrm{C} 2$ & 82 & 32 \\
\hline C3 & 182 & 52 \\
\hline $\mathrm{C} 4$ & 53 & 29 \\
\hline C5 & 327 & 53 \\
\hline C6 & 80 & 39 \\
\hline C7 & 81 & 42 \\
\hline C8 & 302 & 59 \\
\hline C9 & 110 & 38 \\
\hline C10 & 222 & 51 \\
\hline
\end{tabular}

Fig. 8 Expression patterns of differentially expressed TF and TR genes during seed dormancy release and seedling establishment. A Hierarchical clustering. Red and blue represent increased and decreased transcript abundances, respectively. The 10 main subclusters are indicated at the branching points (left side). B Statistics regarding the TF and TR DEGs in Clusters 1-10

identified 1246 DEGs, including 269 TF/TR genes. Among them, 378, 790, and 199 DEGs were associated with $P$. sibiricum MD release (Corm vs Seed), PD release (Str_S vs Corm), and the seedling establishment after the MPD release (Eme vs Str_S) (Table S11, Fig. 9). A hierarchical clustering analysis using the FPKM values of these 1246 DEGs resulted in 12 subclusters (Fig. 9A). The expression of 674 DEGs in Subclusters 1-4 (140 TFs/TRs) was down-regulated mainly in the Corm samples, but was up-regulated in the Str_S samples. The expression levels varied in the Seed and Eme samples. Additionally, we detected 212 down-regulated DEGs in the Corm vs Seed comparison, 390 up-regulated DEGs in the Str_S vs Corm comparison, and 31/57 up/downregulated DEGs in the Eme vs Str_S comparison (Fig. 9B). Subclusters 5-12 (Table S11) contained 572 DEGs (129 TFs/TRs), with up-regulated expression mainly in the Corm samples, but down-regulated expression in the other three samples. Furthermore, 158 up-regulated DEGs in the Corm vs Seed comparison, 398 down- regulated DEGs in the Str_S vs Corm comparison, and 108/25 up/down-regulated DEGs in the Eme vs Str_S comparison were distributed in these eight subclusters (Fig. 9B). Of the DEGs identified by the Corm vs Seed, Str_S vs Corm, and Eme vs Str_S comparisons, only 23 were identified in all three comparisons. We detected 105,444 , and 85 DEGs that were specifically expressed during $P$. sibiricum MD release, PD release, and the seedling establishment after the MPD release, respectively. A Venn diagram analysis revealed 241, 82 and 9 DEGs common to the Corm vs Seed and Str_S vs Corm comparisons, the Str_S vs Corm and Eme vs Str_S comparisons, and the Corm vs Seed and Eme vs Str_S comparisons, respectively (Fig. 9C). The DEGs unique to $P$. sibiricum MD release were distributed in Subclusters 14, and 6-8. In contrast, the DEGs unique to P. sibiricum PD release were distributed in nine subclusters except Subcluster 2, 7, 9. The DEGs unique to the seedling establishment after the MPD release were distributed in Subclusters 2-5, and 7-9 (Fig. 9B). 


\section{A Hierarchical clustering B Statistics}

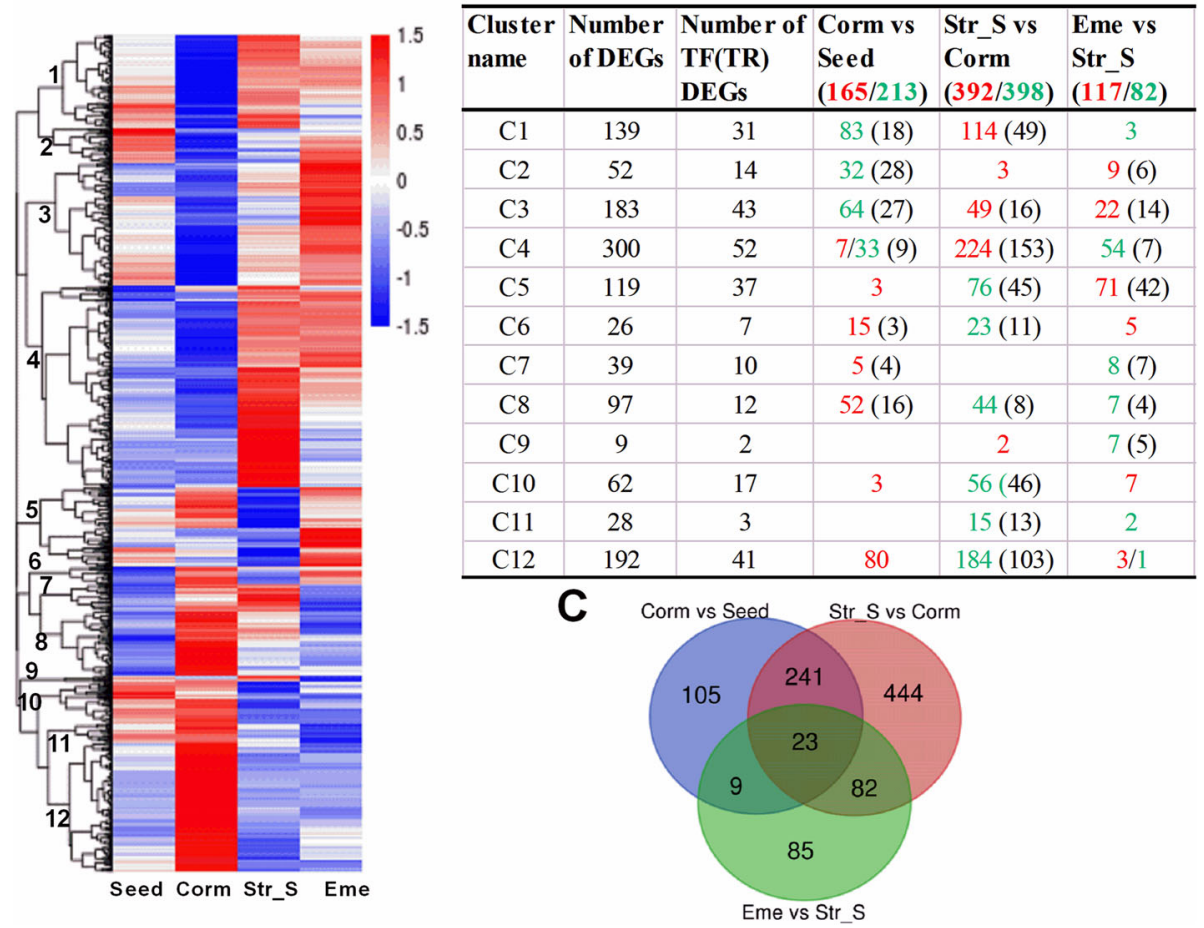

Fig. 9 Expression patterns of differentially expressed seed dormancy-related genes during seed dormancy release and seedling establishment. A Hierarchical clustering. Red and blue represent increased and decreased transcript abundances, respectively. The 12 main subclusters are indicated at the branching points (left side). B Statistics of the DEGs in Clusters 1-12. The numbers of up-regulated DEGs (red) and downregulated DEGs (green) in the Corm vs Seed, Str_S vs Corm, and Eme vs Str_S comparisons are listed in each subcluster. The numbers in parentheses refer to the number of DEGs exclusive to $P$. sibiricum MD release, PD release, and the seedling establishment after the MPD release. $\mathbf{C}$ Venn diagram presenting the number of shared or unique seed dormancy-related genes differentially expressed in the Corm vs Seed, Str_S vs Corm, and Eme vs Str_S comparisons

The known functions of Arabidopsis genes involved in seed dormancy and germination can be classified into several categories, including hormone/light signal transduction, calcium-mediated signaling, chromatin remodeling, mRNA degradation, stress response (AtCYS5), organ development (AtHYL1/AtSE/AtDCL1/ATGSO), and storage matter metabolism (AtATE1/AtATE2/ AtPRT6) (Table 2; Table S11). Subclusters 5-12 included ABA signaling and regulatory genes (e.g., $A B F 1$, $A B I 3, A B I 5$, SIZ1, and SUA), GA signaling genes [e.g., LEUNIG_HOMOLOG (LUH), RGI3, SYL1, and SPY], CK signaling genes (e.g., cytokinin receptor genes $A H K 2$ and AHK4), auxin signaling and responsive genes [AUXIN RESPONSE FACTOR (ARF2), AUXIN RESISTANT1 (AUX1), IAA8, LAX, and PIN], BR signaling genes (BIN2 and BRI1), calcium-mediated signaling genes (e.g., CAM7, CAMAT6, CIPK3, CPK6, and CML39), genes integrating multiple signals [FAR-RED IMPAIRED RESPONSE1 (FAR1), GIGANTEA (GI), MYC2, and TZF1 (tandem zinc finger)], chromatin remodeling genes [e.g., CHR12 (chromatin remodeling ATPase), HAC1 (histone acyltransferase), HDA19 (histone deacetylase), PICKLE (chromatin-remodeling factor), SUVH5 (histone methyltransferase), ROS1 (DNA demethylase), and POWERDRESS (epigenetic factor)], cell wall loosening expansin genes (EXPA4 and EXPA8), and seed storage matter metabolism-related genes [CathB3 (cathepsin B-like cysteine protease), MAN2 (endo- $\beta$-mannanase), MAN7, PROTEOLYSIS6, and ANNAT2 (annexin A2)]. Subclusters 1-4 included genes with the same functions as some of the genes in Subclusters 5-12 (e.g., ARF2 and AHK4) as well as genes that were exclusive to these four subclusters [e.g., AREB3, CORONATINE INSENSITIVE 1, GID1 (GA receptor), and GOLS1 (galactinol synthase)]. The expression of some DEGs that were annotated as the same Arabidopsis homolog or gene family exhibited diverse expression patterns depending on the stage, suggesting they have different roles during $P$. sibiricum MD release, PD release, and the seedling establishment after the MPD release, and are therefore regulated differently. For example, 26 DEGs were annotated as AtWRKY2, 6, 40 , and 41. The expression levels of 11 WRKY TF genes in Subclusters 3 and 5 were all slightly down-regulated during MD release and fluctuated during the cold 
Table 2 Functions and fold changes of selected seed dormany/germination-related DEGs during MD and PD release

\begin{tabular}{|c|c|c|c|c|c|}
\hline Unigene ID & Gene name & Function & $\begin{array}{l}\text { Corm vs } \\
\text { Seed }\end{array}$ & $\begin{array}{l}\text { Str_S vs } \\
\text { Corm }\end{array}$ & $\begin{array}{l}\text { Eme vs } \\
\text { Str_S }\end{array}$ \\
\hline $\begin{array}{l}\text { OO_LQ_SeedM_c3069/ } \\
\text { f1p14/781 }\end{array}$ & AtGIM2 & GA biosynthesis & 1.85 & & \\
\hline $\begin{array}{l}\text { i1_HQ_SeedM_c1802/f5p2/ } \\
1957\end{array}$ & AtNPF3.1 & GA transport & -1.76 & 2.63 & -3.78 \\
\hline $\begin{array}{l}\text { iO_LQ_SeedM_c1919/f2p0/ } \\
830\end{array}$ & AtSLY1 & GA signaling & & -2.23 & \\
\hline $\begin{array}{l}\text { i3_LQ_SeedM_c33971/ } \\
\text { f1p0/3810 }\end{array}$ & AtSPY & GA signaling & -3.75 & 3.98 & \\
\hline $\begin{array}{l}\text { i4_LQ_SeedM_c21157/ } \\
\text { f1p3/4409 }\end{array}$ & AtSPY & GA signaling & 2.66 & -1.96 & \\
\hline $\begin{array}{l}\text { i1_HQ_SeedM_c1756/f2p5/ } \\
1840\end{array}$ & AtGID1C & GA signaling & -1.53 & 2.46 & \\
\hline $\begin{array}{l}\text { i2_LQ_SeedM_c18534/ } \\
\text { f1p7/2257 }\end{array}$ & AtRGI3 & GA signaling & & -1.47 & \\
\hline $\begin{array}{l}\text { i2__HQ_SeedM_c14207/ } \\
\text { f11p9/2283 }\end{array}$ & AtGAl & GA signaling & & -2.8 & \\
\hline $\begin{array}{l}\text { iO_LQ_SeedM_c8549/f1p3/ } \\
650\end{array}$ & AtGASA6 & GA signaling & & -7.4 & 4.14 \\
\hline $\begin{array}{l}\text { i1_LQ_SeedM_c9540/f1p1/ } \\
1818\end{array}$ & AtCYP707A1 & ABA catabolism & 3.28 & -5.32 & \\
\hline $\begin{array}{l}\text { i1_LQ_SeedM_c24968/ } \\
\text { f1p3/1093 }\end{array}$ & AtPYL8 & ABA signaling & & 1.46 & \\
\hline $\begin{array}{l}\text { iO_LQ_SeedM_c8831/f1p3/ } \\
976\end{array}$ & AtPYR1 & ABA signaling & & -2.4 & \\
\hline $\begin{array}{l}\text { i3_LQ_SeedM_c18408/ } \\
\text { f1p3/3496 }\end{array}$ & AtS2P & ABA signaling & 3.3 & -2.78 & \\
\hline $\begin{array}{l}\text { i2_LQ_SeedM_c46616/ } \\
\text { f3p4/2678 }\end{array}$ & AtHAB1 & ABA signaling & -1.67 & 1.43 & \\
\hline $\begin{array}{l}\text { i1_LQ_SeedM_c3858/ } \\
\text { f2p106/1553 }\end{array}$ & AtHON & ABA signaling & -2.52 & 3.13 & \\
\hline $\begin{array}{l}\text { i2_LQ_SeedM_c25671/ } \\
\text { f1p3/2973 }\end{array}$ & AtAFP3 & ABA signaling & -2.4 & 1.94 & \\
\hline $\begin{array}{l}\text { i1_LQ_SeedM_c5687/ } \\
\text { f1p17/2029 }\end{array}$ & AtPP2CA & ABA signaling & & 2.62 & \\
\hline $\begin{array}{l}\text { i1_LQ_SeedM_c9533/f1p0/ } \\
1988\end{array}$ & AtPP2C5 & ABA signaling & -1.81 & & 2.65 \\
\hline $\begin{array}{l}\text { i3_LQ_SeedM_c20125/ } \\
\text { f1p4/3251 }\end{array}$ & AtSnRK2.6 & ABA signaling & & -2.63 & \\
\hline $\begin{array}{l}\text { iO_LQ_SeedM_c10659/ } \\
\text { f1p0/461 }\end{array}$ & AtSnRK3.6 & ABA signaling & & -2.7 & \\
\hline $\begin{array}{l}\text { i5_LQ_SeedM_c2274/f1p2/ } \\
5125\end{array}$ & AtPP2CE & EGR2 phosphatase & -3.34 & 2.56 & \\
\hline $\begin{array}{l}\text { i1_LQ_SeedM_c6124/f1p7/ } \\
1511\end{array}$ & AtHAl3 & ABA signaling & -1.75 & 2.01 & \\
\hline $\begin{array}{l}\text { i4_LQ_SeedM_c21538/ } \\
\text { f1p0/4527 }\end{array}$ & AtABF4 & ABA signaling & & 2.9 & \\
\hline $\begin{array}{l}\text { i1_LQ_SeedM_c8820/f1p3/ } \\
1489\end{array}$ & AtWRKY41 & regulator of $A B \mid 3$ expression & & -2.5 & 4.43 \\
\hline $\begin{array}{l}\text { i2_LQ_SeedM_c36664/ } \\
\text { f1p0/2863 }\end{array}$ & AtABI3 & ABA signaling & & -6.26 & \\
\hline $\begin{array}{l}\text { i1_LQ_SeedM_c22586/ } \\
\text { f1p9/1509 }\end{array}$ & AtABI5 & ABA signaling & & -1.89 & \\
\hline i3_HQ_SeedM_c17466/ & AtARF2 & Auxin signaling & -5.55 & 7.14 & -1.87 \\
\hline
\end{tabular}


Table 2 Functions and fold changes of selected seed dormany/germination-related DEGs during MD and PD release (Continued)

\begin{tabular}{|c|c|c|c|c|c|}
\hline Unigene ID & Gene name & Function & $\begin{array}{l}\text { Corm vs } \\
\text { Seed }\end{array}$ & $\begin{array}{l}\text { Str_S vs } \\
\text { Corm }\end{array}$ & $\begin{array}{l}\text { Eme vs } \\
\text { Str_S }\end{array}$ \\
\hline \multicolumn{6}{|l|}{$\mathrm{f} 2 \mathrm{p} 2 / 3841$} \\
\hline $\begin{array}{l}\text { i3_LQ_SeedM_c7465/ } \\
\text { f1p21/3129 }\end{array}$ & AtARF2 & Auxin signaling & 2.21 & -4.07 & \\
\hline $\begin{array}{l}\text { i0_HQ_SeedM_c12179/ } \\
\text { f2p3/788 }\end{array}$ & AtIAA8 & Auxin signaling & & -1.83 & \\
\hline $\begin{array}{l}\text { i2_LQ_SeedM_c3798/f1p9/ } \\
2925\end{array}$ & AtAUX1 & auxin transport & 4.36 & -3.19 & \\
\hline $\begin{array}{l}\text { i3_LQ_SeedM_c14993/ } \\
\text { f1p0/3065 }\end{array}$ & AtAUX1 & auxin transport & -3.58 & 3.13 & \\
\hline $\begin{array}{l}\text { i2_LQ_SeedM_c41269/ } \\
\text { f1p5/2679 }\end{array}$ & AtLAX & auxin transport & 2.13 & -3.86 & \\
\hline $\begin{array}{l}\text { i4_LQ_SeedM_c20675/ } \\
\text { f1p4/4170 }\end{array}$ & $\begin{array}{l}\text { AtABCB1/ } \\
\text { PGP1 }\end{array}$ & auxin transport & 2.49 & -5.04 & \\
\hline $\begin{array}{l}\text { i4_LQ_SeedM_c4985/f1p0/ } \\
4218\end{array}$ & $\begin{array}{l}\text { AtABCB1/ } \\
\text { PGP1 }\end{array}$ & auxin transport & -4.58 & 3 & \\
\hline $\begin{array}{l}\text { i4_LQ_SeedM_c3741/f1p0/ } \\
4833\end{array}$ & $\begin{array}{l}\text { AtABCB19/ } \\
\text { PGP19 }\end{array}$ & auxin transport & 2.77 & -2.41 & -1.92 \\
\hline $\begin{array}{l}\text { i4_LQ_SeedM_c18157/ } \\
\text { f1p5/4494 }\end{array}$ & AtABCG36 & auxin transport & -3.28 & 6.15 & -3.33 \\
\hline $\begin{array}{l}\text { i2_HQ_SeedM_c17187/ } \\
\text { f5p6/2691 }\end{array}$ & AtPIN1 & auxin transport & -1.61 & 2.1 & \\
\hline $\begin{array}{l}\text { i2_LQ_SeedM_c11796/ } \\
\text { f1p } 4 / 2422\end{array}$ & AtPIN1 & auxin transport & 4.76 & -6.81 & \\
\hline $\begin{array}{l}\text { i2_LQ_SeedM_c37994/ } \\
\text { f1p3/2426 }\end{array}$ & AtPIN1 & auxin transport & & -2.99 & 2.73 \\
\hline $\begin{array}{l}\text { i2_LQ_SeedM_c41403/ } \\
\text { f1p2/2390 }\end{array}$ & AtPIN2 & auxin transport & & -2.9 & 1.88 \\
\hline $\begin{array}{l}\text { i2_LQ_SeedM_c56557/ } \\
\text { f1p2/2726 }\end{array}$ & AtPIN3 & auxin transport & & 1.85 & \\
\hline $\begin{array}{l}\text { i2_LQ_SeedM_c60075/ } \\
\text { f1p3/2081 }\end{array}$ & AtPIN4 & auxin transport & & -5.78 & \\
\hline $\begin{array}{l}\text { i1_HQ_SeedM_c2547/f4p0/ } \\
2003\end{array}$ & AtNRT1.1 & auxin transport & 5.38 & -4.32 & \\
\hline $\begin{array}{l}\text { i2_LQ_SeedM_c37693/ } \\
\text { f1p22/2372 }\end{array}$ & AtBIN2 & BR signaling & -2.05 & 2.97 & \\
\hline $\begin{array}{l}\text { i1_LQ_SeedM_c20577/ } \\
\text { f1p2/1956 }\end{array}$ & AtBIN2 & BR signaling & 5.57 & & \\
\hline $\begin{array}{l}\text { i3_HQ_SeedM_c11682/ } \\
\text { f2p0/3684 }\end{array}$ & AtBRI1 & BR signaling & -6.27 & 5.35 & \\
\hline $\begin{array}{l}\text { i3_LQ_SeedM_c5302/f1p0/ } \\
3667\end{array}$ & AtBRI1 & BR signaling & 5.44 & -6.97 & \\
\hline $\begin{array}{l}\text { i1_LQ_SeedM_c10003/ } \\
\text { f1p34/1668 }\end{array}$ & AtMYC2 & JA signaling & & -1.92 & \\
\hline $\begin{array}{l}\text { i2_LQ_SeedM_c56779/ } \\
\text { f1p3/2496 }\end{array}$ & AtAKIN10 & Energy sensor & -2.81 & 2.76 & \\
\hline $\begin{array}{l}\text { i2_HQ_SeedM_c26414/ } \\
\text { f2p8/2462 }\end{array}$ & AtCOI1 & ubiquitin-dependent protein degradation & & 1.22 & \\
\hline $\begin{array}{l}\text { i0_LQ_SeedM_c9980/f1p0/ } \\
847\end{array}$ & AtASK2 & ubiquitin-dependent protein degradation & -2.8 & 2.15 & \\
\hline $\begin{array}{l}\text { i0_LQ_SeedM_c3002/f1p6/ } \\
571\end{array}$ & AtRHA2b & ubiquitin-dependent protein degradation & & -2.56 & \\
\hline $\begin{array}{l}\text { i3_HQ_SeedM_c7511/ } \\
\text { f2p11/3746 }\end{array}$ & AtUBP26 & ubiquitin-dependent protein degradation & 2.92 & -2.44 & \\
\hline
\end{tabular}


Table 2 Functions and fold changes of selected seed dormany/germination-related DEGs during MD and PD release (Continued)

\begin{tabular}{|c|c|c|c|c|c|}
\hline Unigene ID & Gene name & Function & $\begin{array}{l}\text { Corm vs } \\
\text { Seed }\end{array}$ & $\begin{array}{l}\text { Str_S vs } \\
\text { Corm }\end{array}$ & $\begin{array}{l}\text { Eme vs } \\
\text { Str_S }\end{array}$ \\
\hline $\begin{array}{l}\text { i2_LQ_SeedM_c48471/ } \\
\text { f2p3/2487 }\end{array}$ & AtATE2 & protein degradation & & -1.91 & \\
\hline $\begin{array}{l}\text { i4_HQ_SeedM_c15243/ } \\
\text { f2p0/4126 }\end{array}$ & AtCOP10 & ubiquitin-conjugating enzyme E2 & & -1.58 & \\
\hline $\begin{array}{l}\text { i3_LQ_SeedM_c15448/ } \\
\text { f1p3/3019 }\end{array}$ & AtDWA & $\begin{array}{l}\text { substrate receptor for cullin-RING ubiquitin ligase } 4 \text { complexes for } \\
\text { protein degradation }\end{array}$ & -2.11 & 1.95 & \\
\hline $\begin{array}{l}\text { iO_LQ_SeedM_c5286/f1p0/ } \\
992\end{array}$ & AtCathB3 & Cysteine proteinase & 4 & -4.3 & \\
\hline $\begin{array}{l}\text { i3_LQ_SeedM_c32927/ } \\
\text { f1p20/3736 }\end{array}$ & AtSIZ1 & protein sumoylation & 3.27 & -4.75 & \\
\hline $\begin{array}{l}\text { i3_LQ_SeedM_c22466/ } \\
\text { f1p6/3768 }\end{array}$ & AtSIZ1 & protein sumoylation & -1.89 & 2.66 & \\
\hline $\begin{array}{l}\text { i2_LQ_SeedM_c11836/ } \\
\text { f4p3/2198 }\end{array}$ & AtKAPP & kinase-associated protein phosphatase & 3.34 & -4.77 & \\
\hline $\begin{array}{l}\text { i2_LQ_SeedM_c56819/ } \\
\text { f1p3/2379 }\end{array}$ & AtKAPP & kinase-associated protein phosphatase & -4.31 & 3.14 & \\
\hline $\begin{array}{l}\text { i1_LQ_SeedM_c7267/ } \\
\text { f1p11/1962 }\end{array}$ & AtDjA3 & Chaperone protein & -3.28 & 2.05 & \\
\hline $\begin{array}{l}\text { i2_LQ_SeedM_c66006/ } \\
\text { f1p10/2500 }\end{array}$ & AtDjA3 & Chaperone protein & 2.34 & -2.54 & \\
\hline $\begin{array}{l}\text { iO_LQ_SeedM_c17946/ } \\
\text { f1p1/823 }\end{array}$ & AtCAM7 & calcium signaling & 1.76 & -3.25 & \\
\hline $\begin{array}{l}\text { i4_LQ_SeedM_c16211/ } \\
\text { f1p0/4095 }\end{array}$ & AtCAM7 & calcium signaling & -4.25 & 3.63 & \\
\hline $\begin{array}{l}\text { i1_LQ_SeedM_c15573/ } \\
\text { f1p3/1075 }\end{array}$ & AtCML39 & calcium signaling & & -4.27 & 6.47 \\
\hline $\begin{array}{l}\text { i4_LQ_SeedM_c16133/ } \\
\text { f1p0/4048 }\end{array}$ & AtCAMTA6 & calmodulin-binding TF & & 3.78 & \\
\hline $\begin{array}{l}\text { i3_LQ_SeedM_c4760/f1p1/ } \\
3504\end{array}$ & AtCAMTA6 & calmodulin-binding TF & & -2.51 & \\
\hline $\begin{array}{l}\text { i2_LQ_SeedM_c52294/ } \\
\text { f1p1/2157 }\end{array}$ & AtlQM4 & $\mathrm{Ca} 2+-$ independent CaM-binding protein & & 1.69 & \\
\hline $\begin{array}{l}\text { i2_LQ_SeedM_c59734/ } \\
\text { f2p3/2262 }\end{array}$ & AtCPK6 & Calcium-dependent protein kinase & 4.13 & -3.16 & \\
\hline $\begin{array}{l}\text { i3_LQ_SeedM_c18100/ } \\
\text { f1p6/3246 }\end{array}$ & AtGLR3.5 & ionotropic glutamate receptor & -1.9 & & \\
\hline $\begin{array}{l}\text { i1_LQ_SeedM_c21031/ } \\
\text { f1p0/1778 }\end{array}$ & AtCAT1 & catalase & 4.88 & -7.79 & 4.03 \\
\hline $\begin{array}{l}\text { i1_LQ_SeedM_c5525/f1 p5/ } \\
1792\end{array}$ & AtTFIIS & transcript elongation factor & & -1.59 & 1.53 \\
\hline $\begin{array}{l}\text { i2_HQ_SeedM_c3213/f3p5/ } \\
2849\end{array}$ & AtCBC80 & RNA metabolism & -3.02 & & \\
\hline $\begin{array}{l}\text { i2_LQ_SeedM_c13505/ } \\
\text { f1p1/2439 }\end{array}$ & AtCFM9 & RNA metabolism & & -1.82 & \\
\hline $\begin{array}{l}\text { i6_LQ_SeedM_c1081/f1p4/ } \\
6064\end{array}$ & AtDCL1 & RNA metabolism & 2.82 & -2.07 & -2.58 \\
\hline $\begin{array}{l}\text { i2_LQ_SeedM_c23333/ } \\
\text { f1p14/2786 }\end{array}$ & AtSE & RNA metabolism & -2.41 & 2.59 & \\
\hline $\begin{array}{l}\text { i2_LQ_SeedM_c27773/ } \\
\text { f1p0/2851 }\end{array}$ & AtNG1 & RNA metabolism & & 2.71 & \\
\hline $\begin{array}{l}\text { iO_LQ_SeedM_c2368/f1p9/ } \\
737\end{array}$ & AtGRP7 & RNA metabolism & -4 & 3.68 & \\
\hline i4_HQ_SeedM_c3551/f2p0/ & AtSUA & RNA metabolism & -6.6 & 5.58 & \\
\hline
\end{tabular}


Table 2 Functions and fold changes of selected seed dormany/germination-related DEGs during MD and PD release (Continued)

\begin{tabular}{|c|c|c|c|c|c|}
\hline Unigene ID & Gene name & Function & $\begin{array}{l}\text { Corm vs } \\
\text { Seed }\end{array}$ & $\begin{array}{l}\text { Str_S vs } \\
\text { Corm }\end{array}$ & $\begin{array}{l}\text { Eme vs } \\
\text { Str_S }\end{array}$ \\
\hline \multicolumn{6}{|l|}{4808} \\
\hline $\begin{array}{l}\text { i3_LQ_SeedM_c23460/ } \\
\text { f1p11/3454 }\end{array}$ & AtSUA & RNA metabolism & 6.77 & -6.94 & \\
\hline $\begin{array}{l}\text { i2_LQ_SeedM_c23171/ } \\
\text { f1p11/2948 }\end{array}$ & AtFY & mRNA processing & 2.17 & -2.07 & \\
\hline $\begin{array}{l}\text { i3_LQ_SeedM_c20086/ } \\
\text { f1p0/3799 }\end{array}$ & AtFY & mRNA processing & -4.59 & 4.24 & \\
\hline $\begin{array}{l}\text { i3_LQ_SeedM_c30639/ } \\
\text { f1p0/3050 }\end{array}$ & AtSF1 & mRNA processing & 6.59 & -7.57 & \\
\hline $\begin{array}{l}\text { i3_LQ_SeedM_c9329/f1p0/ } \\
3408\end{array}$ & AtSF1 & mRNA processing & & 6.55 & \\
\hline $\begin{array}{l}\text { i1_LQ_SeedM_c4043/f1p9/ } \\
1960\end{array}$ & AtRRP41L & mRNA degradation & & 1.74 & \\
\hline $\begin{array}{l}\text { i3_LQ_SeedM_c9561/ } \\
\text { f1p61/3436 }\end{array}$ & AtXRN4 & mRNA degradation & 4.36 & -6.43 & \\
\hline $\begin{array}{l}\text { i5_LQ_SeedM_c4155/f1p1/ } \\
5195\end{array}$ & AtXRN4 & mRNA degradation & & 5.44 & -5.8 \\
\hline $\begin{array}{l}\text { i4_LQ_SeedM_c2767/f1p0/ } \\
4467\end{array}$ & AtVCS & mRNA degradation & -3.54 & 2.82 & \\
\hline $\begin{array}{l}\text { i3_LQ_SeedM_c9026/f1p0/ } \\
3591\end{array}$ & AtVCS & mRNA degradation & 1.95 & -2.55 & \\
\hline $\begin{array}{l}\text { i2_LQ_SeedM_c10778/ } \\
\text { f1p19/2311 }\end{array}$ & AtTudor2 & mRNA degradation & -1.55 & 1.58 & \\
\hline $\begin{array}{l}\text { i1_LQ_SeedM_c2372/ } \\
\text { f1p11/1714 }\end{array}$ & AtCHO1 & AP2/ERF-AP2 TF & & -2.13 & \\
\hline $\begin{array}{l}\text { i2_LQ_SeedM_c29982/ } \\
\text { f1p4/2011 }\end{array}$ & AtASG1 & DNA helicase & & -1.93 & \\
\hline $\begin{array}{l}\text { i2_LQ_SeedM_c4618/f1p3/ } \\
2576\end{array}$ & AtASG1 & DNA helicase & & 2.39 & \\
\hline $\begin{array}{l}\text { i4_LQ_SeedM_c25313/ } \\
\text { f1p14/4107 }\end{array}$ & AtEMF1 & histone methylation & -2.76 & 2.38 & \\
\hline $\begin{array}{l}\text { i5_LQ_SeedM_c10657/ } \\
\text { f1p1/5263 }\end{array}$ & AtCHR12 & chromatin modification & 3.6 & -3.5 & \\
\hline $\begin{array}{l}\text { i3_LQ_SeedM_c6562/f1p0/ } \\
3365\end{array}$ & AtCLF & chromatin modification & -1.98 & 2.68 & \\
\hline $\begin{array}{l}\text { i6_LQ_SeedM_c990/f1p0/ } \\
6104\end{array}$ & AtEFS & chromatin modification & & -3.15 & \\
\hline $\begin{array}{l}\text { i5_HQ_SeedM_c466/f6p0/ } \\
6094\end{array}$ & AtHACl & chromatin modification & -2.19 & 1.83 & \\
\hline $\begin{array}{l}\text { i4_LQ_SeedM_c1879/f1p8/ } \\
5031\end{array}$ & AtHAC1 & chromatin modification & 2.98 & -2.54 & \\
\hline $\begin{array}{l}\text { i5_LQ_SeedM_c8676/f1p0/ } \\
5895\end{array}$ & AtPKL & chromatin modification & -8.64 & 7.42 & 2.2 \\
\hline $\begin{array}{l}\text { i4_LQ_SeedM_c13881/ } \\
\text { f1p0/4335 }\end{array}$ & AtPKL & chromatin modification & 1.98 & -2.06 & 2.01 \\
\hline $\begin{array}{l}\text { i3_LQ_SeedM_c14311/ } \\
\text { f1p0/3164 }\end{array}$ & AtSUVH5 & chromatin modification & & -4.1 & 2.64 \\
\hline $\begin{array}{l}\text { i3_LQ_SeedM_c6655/f1p1/ } \\
3369\end{array}$ & AtHDA9 & chromatin modification & & 4.93 & 2.32 \\
\hline $\begin{array}{l}\text { i2_LQ_SeedM_c6979/f1p2/ } \\
2448\end{array}$ & AtHDA15 & chromatin modification & & -1.56 & \\
\hline $\begin{array}{l}\text { i4_LQ_SeedM_c18037/ } \\
\text { f1p21/4801 }\end{array}$ & AtPWR & chromatin modification & 1.93 & -2.1 & 1.61 \\
\hline
\end{tabular}


Table 2 Functions and fold changes of selected seed dormany/germination-related DEGs during MD and PD release (Continued)

\begin{tabular}{|c|c|c|c|c|c|}
\hline Unigene ID & Gene name & Function & $\begin{array}{l}\text { Corm vs } \\
\text { Seed }\end{array}$ & $\begin{array}{l}\text { Str_S vs } \\
\text { Corm }\end{array}$ & $\begin{array}{l}\text { Eme vs } \\
\text { Str_S }\end{array}$ \\
\hline $\begin{array}{l}\text { i2_LQ_SeedM_c27191/ } \\
\text { f1p4/2756 }\end{array}$ & AtCRY1 & light signaling & 3.66 & -3.76 & \\
\hline $\begin{array}{l}\text { i2_HQ_SeedM_c35624/ } \\
\text { f2p2/2375 }\end{array}$ & AtCRY2 & light signaling & -2.16 & 3.56 & \\
\hline $\begin{array}{l}\text { i3_LQ_SeedM_c33308/ } \\
\text { f1p0/3596 }\end{array}$ & AtPHYA & light signaling & 2.9 & -4.08 & \\
\hline $\begin{array}{l}\text { i2_LQ_SeedM_c36242/ } \\
\text { f1p4/2456 }\end{array}$ & AtPIF3 & light and GA signaling & -5.16 & 4.2 & \\
\hline $\begin{array}{l}\text { i2_LQ_SeedM_c21496/ } \\
\text { f1p3/2439 }\end{array}$ & AtPIF3 & light and GA signaling & & -2.43 & \\
\hline $\begin{array}{l}\text { i1_LQ_SeedM_c17359/ } \\
\text { f1p1/1778 }\end{array}$ & AtCIPK3 & CBL-interacting protein kinase & -2.74 & 2.91 & -2.3 \\
\hline $\begin{array}{l}\text { i3_LQ_SeedM_c21126/ } \\
\text { f1p9/3152 }\end{array}$ & AtPRR9 & circadian rhythm & -7.9 & 6.9 & \\
\hline $\begin{array}{l}\text { i2_LQ_SeedM_c52693/ } \\
\text { f1p0/2189 }\end{array}$ & AtTOC1 & circadian rhythm & & 1.7 & \\
\hline $\begin{array}{l}\text { i2_LQ_SeedM_c48681/ } \\
\text { f1p } 5 / 2590\end{array}$ & AtMED25 & light and JA signaling & 1.98 & -1.94 & \\
\hline $\begin{array}{l}\text { i1_LQ_SeedM_c10304/ } \\
\text { f1p0/1883 }\end{array}$ & AtCYCD1;1 & cell cycle protein & & -2.59 & 1.73 \\
\hline $\begin{array}{l}\text { i2_LQ_SeedM_c66356/ } \\
\text { f1p3/2916 }\end{array}$ & AtSAUR62 & Cell-elongated related genes & & 2.01 & \\
\hline $\begin{array}{l}\text { i1_HQ_SeedM_c2317/f3p4/ } \\
1843\end{array}$ & AtWRKY2 & WRKY TF & 3.07 & -10.19 & \\
\hline $\begin{array}{l}\text { i2_LQ_SeedM_c69494/ } \\
\text { f1p7/2362 }\end{array}$ & AtWRKY2 & WRKY TF & -2.1 & 3.34 & \\
\hline $\begin{array}{l}\text { i2_LQ_SeedM_c59966/ } \\
\text { f1p0/2048 }\end{array}$ & AtWRKY6 & WRKY TF & & 4.72 & \\
\hline $\begin{array}{l}\text { i2_LQ_SeedM_c50026/ } \\
\text { f1p2/2377 }\end{array}$ & AtWRKY40 & WRKY TF & & -2.47 & 4.13 \\
\hline $\begin{array}{l}\text { i3_LQ_SeedM_c23462/ } \\
\text { f1p0/3258 }\end{array}$ & AtGBF1 & bZIP TF & & 2.1 & \\
\hline $\begin{array}{l}\text { i2_LQ_SeedM_c36125/ } \\
\text { f1p5/3079 }\end{array}$ & AtLUG & transcriptional corepressor & 2.2 & -5.54 & \\
\hline $\begin{array}{l}\text { i3_LQ_SeedM_c29344/ } \\
\text { f1p0/3137 }\end{array}$ & AtLUG & transcriptional corepressor & -3.02 & 3.29 & \\
\hline $\begin{array}{l}\text { i2_LQ_SeedM_c21274/ } \\
\text { f1p0/2367 }\end{array}$ & AtNIA1 & nitrate assimilation & 6.39 & -7.04 & \\
\hline $\begin{array}{l}\text { i3_LQ_SeedM_c30611/ } \\
\text { f1p0/3119 }\end{array}$ & AtGCN2 & amino acid metabolism & -7.11 & 6.39 & \\
\hline $\begin{array}{l}\text { i1_LQ_SeedM_c17195/ } \\
\text { f2p0/1544 }\end{array}$ & AtGln $1 ; 1$ & amino acid metabolism & 1.47 & -1.53 & \\
\hline $\begin{array}{l}\text { i3_LQ_SeedM_c12339/ } \\
\text { f1p3/3098 }\end{array}$ & AtTPS5 & trehalose biosynthesis & -1.89 & & \\
\hline $\begin{array}{l}\text { i1_LQ_SeedM_c20939/ } \\
\text { f1p0/1223 }\end{array}$ & AtGOLS1 & galactinol synthase & & 4.59 & -1.69 \\
\hline $\begin{array}{l}\text { i2_HQ_SeedM_c62143/ } \\
\text { f13p14/2507 }\end{array}$ & AtMFP2 & Fatty acid degradation & 3.92 & -4.32 & \\
\hline $\begin{array}{l}\text { i2_LQ_SeedM_c18604/ } \\
\text { f1p14/2485 }\end{array}$ & AtMFP2 & Fatty acid degradation & & 3.99 & \\
\hline $\begin{array}{l}\text { i1_LQ_SeedM_c11103/ } \\
\text { f1p4/1439 }\end{array}$ & AtLIP1 & lipid metabolism & 2.62 & -1.9 & 1.69 \\
\hline i3_LQ_SeedM_c26493/ & AtKPNB1 & protein transport & & -1.58 & \\
\hline
\end{tabular}


Table 2 Functions and fold changes of selected seed dormany/germination-related DEGs during MD and PD release (Continued)

\begin{tabular}{|c|c|c|c|c|c|}
\hline Unigene ID & Gene name & Function & $\begin{array}{l}\text { Corm vs } \\
\text { Seed }\end{array}$ & $\begin{array}{l}\text { Str_S vs } \\
\text { Corm }\end{array}$ & $\begin{array}{l}\text { Eme vs } \\
\text { Str_S }\end{array}$ \\
\hline \multicolumn{6}{|l|}{$f 1 p 7 / 3651$} \\
\hline $\begin{array}{l}\text { i3_LQ_SeedM_c30926/ } \\
\text { f1p0/3119 }\end{array}$ & AtPHO1 & phosphate ion transport & & -4.63 & \\
\hline $\begin{array}{l}\text { i2_LQ_SeedM_c20018/ } \\
\text { f1p30/2524 }\end{array}$ & AtCER9 & cuticular wax biosynthesis & -3.68 & & 2.14 \\
\hline $\begin{array}{l}\text { i1_LQ_SeedM_c5889/f1p2/ } \\
1648\end{array}$ & AtPGIP2 & cell wall metabolism & & -1.6 & \\
\hline $\begin{array}{l}\text { i1_LQ_SeedM_c8885/f1p0/ } \\
1852\end{array}$ & AtPLY & cell wall metabolism & 2.57 & -5.77 & \\
\hline $\begin{array}{l}\text { i1_LQ_SeedM_c22277/ } \\
\text { f1p18/1916 }\end{array}$ & AtPME58 & cell wall metabolism & & 5.59 & -4.03 \\
\hline $\begin{array}{l}\text { i1_LQ_SeedM_c20327/ } \\
\text { f1p9/1915 }\end{array}$ & AtMAN2 & cell wall metabolism & & -8.51 & 5.39 \\
\hline $\begin{array}{l}\text { i2_LQ_SeedM_c17702/ } \\
\text { f1p2/2902 }\end{array}$ & AtMAN7 & cell wall metabolism & 3.28 & -4.5 & \\
\hline $\begin{array}{l}\text { i1_LQ_SeedM_c15435/ } \\
\text { f1p3/1132 }\end{array}$ & AtEXPA4 & cell wall metabolism & 1.58 & -3.82 & 1.66 \\
\hline $\begin{array}{l}\text { i1_LQ_SeedM_c2665/f1p0/ } \\
2002\end{array}$ & AtNEK6 & cortical microtubule organization & & -1.92 & \\
\hline $\begin{array}{l}\text { i2_LQ_SeedM_c6535/f2p1/ } \\
2253\end{array}$ & AtDREB2C & DREB TF & & 1.73 & \\
\hline $\begin{array}{l}\text { i2_LQ_SeedM_c3709/f1p3/ } \\
2689\end{array}$ & AtERD15 & dehydration-induced protein & -3.37 & 1.92 & \\
\hline $\begin{array}{l}\text { i1_LQ_SeedM_c22084/ } \\
\text { f1p0/1316 }\end{array}$ & AtLEA31 & Seed maturation protein & & 4.12 & -1.77 \\
\hline $\begin{array}{l}\text { i4_LQ_SeedM_c19775/ } \\
\text { f1p33/4207 }\end{array}$ & AtGSO1 & peptide hormone signaling & & -2.57 & \\
\hline
\end{tabular}

Note: Gene was named based on annotated Arabidopsis homologs. The full information of gene regulating Arabidopsis seed dormancy and germination was given in Table S11

continues to grow and differentiate, which is accompanied by the emergence of a plumule

stratification; however, the expression levels of all of these genes were significantly up-regulated at the seedling establishment stage (relative to the expression levels in the MPD seeds). The expression of 15 DEGs annotated as AtWRKY2 in Subclusters 4 (1), 8 (1), 10 (4), 11 (1), and 12 (5) exhibited distinct changing trends during MPD release and seedling establishment. The expression of AtWRKY6, which encodes a positive regulator of ABA signaling during seed germination and early seedling development, is reportedly repressed during seed germination [24], but it is substantially up-regulated in Arabidopsis germinated seeds under light [25] (http:// bar.utoronto.ca/efp/cgi-bin/efpWeb.cgi). However, AtWRKY2 mediates ABA-dependent seed germination and early postgermination growth [26], and AtWRKY2 expression is up-regulated considerably by exogenous ABA [26] and in germinated seeds under light [25] (http://bar.utoronto.ca/efp/cgi-bin/efpWeb.cgi). In our study, most P. sibiricum homologs of AtWRKY2 were slightly or significantly more highly expressed in the corm than in the seed, but the expression levels decreased during PD release and seedling emergence, which may be closely related to the simultaneous decrease in the expression of its regulators ABI5 and ABI3 [26].

\section{Discussion}

Polygonatum sibiricum is a well-known traditional Chinese medicinal plant throughout east Asian countries. Studies regarding the cultivation and seed biology of this plant species have been conducted, but its seed germination characteristics remain unclear [17, 27-30]. Cheng et al. and $\mathrm{Zhu}$ et al. reported that $\mathrm{GA}_{3}$ and a lowtemperature treatment $\left(0^{\circ} \mathrm{C}\right.$ for 120 days $)$ may enhance P. sibiricum seed germination $[28,30]$. However, our research [12] suggested that warm stratification followed by a low-temperature stratification is a more appropriate strategy for inducing $P$. sibiricum seed germination, which accords with the characteristics of the seeds with deep simple epicotyl MPD [31]. Under natural conditions, $P$. sibiricum mature seeds are dispersed in mid-fall and germinate under suitable warm conditions during the following spring and summer, with only the radicle and corm emerging. The shoot emerges in the spring 
after the second cold winter, indicating that $P$. sibiricum seeds exhibit the deep morphophysiological epicotyl dormancy. However, P. sibiricum seeds can develop into seedlings within 6 months following the successive exposures to warm/cold conditions [12]. Polygonatum kingianum, another important source of polygonati rhizoma, is mainly grown in Yunnan province, China. Its mature seed has an underdeveloped embryo and possesses the similar seed germination characteristics to $P$. sibiricum [32]. However, they had the distinct seedling establishment processes after corm formation. No P. sibiricum seedling emerged under prolonged warm conditions after corm formation, indicating that a cold treatment is essential for the epicotyl dormancy release of $P$. sibiricum corm and its leaf emergence. However, P. kingianum germinated seeds had about $50 \%$ seedling emergence when inculated at $25^{\circ} \mathrm{C}$ [33]. In view of this, an additional low-temperature treatment seems unnecessary for $P$. kingianum seedling emergence after corm formation, although an appropriate chilling period can enhance the emergence rate of $P$. kingianum seedling and accelerate its emergence [32, 33]. These results indicated that Polygonatum species growing in different climatic regions have distinct seed germination properties and regulatory mechanisms.

To understand the molecular mechanisms of corm formation under warm temperature, cold stratification to break the epicotyl dormancy, and seedling establishment during $P$. sibiricum seed germination, we performed gene expression analyses of four stages (Seed, Corm, Str_S, and Eme) using transcriptome sequencing techniques. During the MD release, $P$. sibiricum seeds stratified at warm temperature undergo several important morphological changes including embryo growth, radicle emergence and elongation, and formation of a plumule-containing cormlet. Our DEG analyses revealed that there were more DEGs in the Corm vs Str_S and Corm vs Eme comparisons than in the other comparisons, implying that corm development is a key stage for the transcriptional regulation of $P$. sibiricum seed germination and seedling emergence. It also indicated that a specific cold stratification treatment period breaks the epicotyl dormancy via the expression-level changes of many genes in the developed corm. Fewer DEGs between the mature seed and the corm were identified during MD release. This may have been because our sampling time-points with the bulk seed did not cover all the important developmental events occurring in embryo differentiation and endosperm weakening during MD release, given that the genes determining plant development and growth are usually spatially and temporally expressed [34, 35]. Wang et al. [32] found that 7 TFs including DAG2, Dof5.7, bZIP60, MYB111, MYB55, $M Y B 46$, and REM1 possibly regulated the expression of
17 hub genes that were altered among three different dormant statuses of $P$. kingianum corm. However, we only detected three homologs of AtbZIP60 in our experimental condition and found that they (c18106, c17463 and c8065) were all decreased during cold stratification and elevated in Corm and Eme under warm temperature (Table S9). bZIP60 TF has been found to modulate the unfolded protein response (UPR) in plants and could enhance heat stress tolerance [36]. The upregulation of bZIP60 transcripts in P. sibiricum corm and seedling under warm temperature may suggest its role of warm temperature tolerance.

Studies on plant species with MPD seeds [37-40] revealed that their seed dormancy and germination is controlled jointly by endogenous hormones and environmental conditions including temperature, soil or seed moisture, light, smoke, and nutrient availability, which is similar to Arabidopsis and cereal crops with physiological dormant seeds [41-45]. In addition to ABA and GA, which are two major hormones that respectively induce and break seed dormancy in most plants, other phytohormones, such as cytokinins, jasmonic acid, strigolactones, brassinosteroids, ethylene, salicylic acid, and auxin, may also regulate seed dormancy and germination in a plant species-dependent manner. Their contents in dormant and germinating seeds are partly regulated by the expression of genes related to hormone metabolism and signaling $[40,46]$. In this study, we identified 475 putative hormone metabolismrelated unigenes and 510 putative hormone signaling genes that were differentially expressed during $P$. sibiricum seed dormancy and seedling and shoot emergence (Figs. 6 and 7, Figure S6). Although ABA is considered to be crucial for inducing and maintaining seed dormancy, most $P$. sibiricum DEGs regulating ABA biosynthesis and degradation such as NPQ1, ABA2, CYP707A1 were more highly expressed in the Corm, Str_S, and Eme stages than in the Seed stage. $A B I 3$ and $A B I 5$ are two ABA signaling-related genes that positively control seed dormancy [43]. ABI5 is found highly expressed in dormant seeds of several plant species $[19,46]$. Their homologous genes in $P$. sibiricum were expressed at higher levels in the Seed and Corm stages than in the cold-stratified germinated seeds and seedlings, implying ABA may have accumulated during the Seed and Corm stages under warm conditions, leading to the dormancy of the Seed and Corm stages. In P. cyrtonema, which has similar seed dormancy/germination characteristics to $P$. sibiricum, the ABA level in seeds stored for a long period in wet sand is reportedly higher under warm conditions than under cold conditions [40]. It was also found that $P$. kingianum germinated seeds at corm stage had the higher ABA content than cold-stratified and non-dormant germinated seeds, which was consistent 
with the higher expression of $\mathrm{ABA}$ synthesis-related transcripts [33]. Fluridone is an inhibitor of ABA biosynthesis and promoted seed dormancy release like cold stratification $[47,48]$. In our recent pre-experiments, we observed that fluridone-treated $P$. sibiricum seeds germinate at a higher rate than untreated seeds (data not shown). Hence, a fluridone treatment of $P$. sibiricum MD seeds and the corm after MD release may be useful for elucidating the effects of ABA on P. sibiricum seed $M D$ release and the induction of epicotyl physiological dormancy under warm conditions as well as epicotyl dormancy under cold conditions.

Consistent with molecular regulation of PD seed germination of Arabidopsis [43], many genes related to chromatin modifiers and remodelers, DNA methylation, mRNA degradation, and cell wall structures were also differentially regulated during $P$. sibiricum seed germination, epicotyl dormancy release, and seedling establishment (Table 2, Fig. 9, Table S11). During the germination of $P$. polyphylla, $P$. quinquefolius, and $P$. suffruticosa seeds, the cotyledons remain inside the seed coat/endosperm after MD release and have to be pulled outside until the endosperm is sufficiently weakened to eliminate the mechanical resistance from the surrounding tissues (testa and endosperm). In contrast, the plumule of P. sibiricum develops outside of the hard and compact endosperm after MD release. However, the plumule does not immediately differentiate and elongate to push the shoot out, and this epicotyl physiological dormancy may be correlated with inhibitors in the corm [12] and the slow mobilization and transport of endospermic reserves. Seed storage matter metabolismrelated genes, such as CathB3, MAN2, MAN7, PROTEOLYSIS6, and ANNAT2, as well as cell wall loosening genes (EXPA4 and EXPA8) and polygalacturonase genes (e.g. c19523, c4968) were highly expressed in the corm and then decreased considerably during the cold stratification and seedling emergence. The $\beta$-mannanase and polygalacturonase activities in $P$. sibiricum seeds increase as the endosperm weakens and seed germination proceeds during a warm stratification [49].

\section{Conclusions}

In summary, we analyzed seed samples at four key stratification stages to explore the molecular mechanism regulating seed germination and dormancy release. A full-length transcriptome database was established, after which the expression patterns of some dormancy-related DEGs at the Corm, Str_S, and other stages were analyzed. The results of this study have helped to further characterize the $P$. sibiricum seed dormancy trait and may form the basis of future related investigations. Specifically, we built databases comprising unigenes involved in the $P$. sibiricum seed germination process and phytohormone-related unigenes associated with seed dormancy (Table S8 and Table S11). These databases may enable researchers to further elucidate the molecular mechanism underlying seed dormancy and germination.

\section{Methods}

\section{Plant materials}

Polygonatum sibiricum Red plants are originated from Qishan County, Shanxi province, China and kindly provided by a local $P$. sibiricum growing farmer. They were identified by Dr. Jianjun Qi at the Institute of Medicinal Plant Development. These plants were then cultivated and conserved in a shaded field at the Institute of Medicinal Plant Development, Beijing, China. The seed voucher specimen (HGJG0012) was deposited at the national medicinal plant gene bank of the Institute of Medicinal Plant Development, Beijing, China. The normal field management was applied, according to our institutional field plantation guidelines. Yellow or black ripe berries were collected in early October 2016, fermented at room temperature for several days to soften the pulp outside the seeds, and then rubbed with a fine nylon mesh bag to obtain clean seeds. Fully-filled and healthy mature seeds were soaked in tap water for 1 day and then stratified in moist sand in a plastic box (10 $\mathrm{X} 15 \mathrm{~cm}$ ), which was covered with a lid to delay evaporation. Three sequential temperature stratifications were used to induce $P$. sibiricum seed germination and seedling emergence: 1) warm stratification at $25^{\circ} \mathrm{C}$ for $4-6$ weeks to promote radicle extrusion and cormlet formation; 2) germinated seeds with corms were subjected to a cold stratification at $4^{\circ} \mathrm{C}$ for 8 weeks; 3) the coldstratified seeds were transferred to $25^{\circ} \mathrm{C}$ to induce seedling emergence. Warm and cold stratifications were conducted separately in a temperature-controlled incubator and in a refrigerator. In this study, the following P. sibiricum samples were collected separately: mature seeds soaked for 1 day at room temperature before the $25^{\circ} \mathrm{C}$ stratification (Seed); seeds at the early germination stage during a warm stratification, with an extruded radicle (Ger-S); germinated seeds with a corm during a warm stratification (Corm); seeds at the early stage (about 4 weeks) of a cold stratification (Str), seeds at the late stage (about 8 weeks) of a cold stratification (Str_S); and seeds at the seedling emergence stage during a warm stratification (Eme). The samples were frozen with liquid nitrogen and then stored at $-80^{\circ} \mathrm{C}$ for later use.

Iso-seq library construction and PACBIO SMRT sequencing To well represent the transcriptome information from different seed stages, we sequenced the full-length of the expressed genes in seeds of different stages (Seed, Ger-S, Corm, Str, Str_S, and Eme) using the PacBio technique. 
The work pipeline of our experiment to generate Iso-seq and RNA-seq data was shown in Figure S9. Total RNA was extracted from the collected $P$. sibiricum seeds (Seed, Ger-S, Corm, Str, Str_S, and Eme) using the TRIzol reagent (Invitrogen, USA) according to the manufacturer's instructions. RNA samples with RIN values $\geq 7.8$ were mixed equally to form an RNA pool for constructing the Iso-Seq library and the PacBio full-length cDNA sequencing. Poly-(A) RNA was isolated from the RNA pool using the oligo-(dT) magnetic bead-binding method and the Poly-(A) Purist ${ }^{\mathrm{TM}}$ Kit (Invitrogen, USA). The isolated poly-(A) RNA was eluted with RNase-free water. The mRNA $(1 \mu \mathrm{g})$ was used as the template to synthesize cDNA with the Clontech SMARTer cDNA synthesis kit. After the PCR amplification, quality control, and purification steps were completed, the BluePippin Size Selection System was used to produce three fractions containing fragments $1-2,2-3$, and $3-6 \mathrm{~kb}$ long. The cDNA products were then used to construct SMRTBell Template libraries using the SMRTBell Template Prep Kit. The concentration and quality of the cDNA library were determined using the Qubit 2.0 fluorometer and the Agilent 2100 Bioanalyzer, respectively. All the operations during the library construction followed the protocols of the above-used kits. Finally, three SMRT cells were sequenced on the PacBio RS platform (Pacific Biosciences, Menlo Park, CA, USA) at Beijing Novogene Scientific Co., Ltd. (Beijing, China).

\section{RNA-seq library construction and sequencing}

Samples collected at the Seed, Corm, Str_S, and Eme stages (three biological replicates) underwent an RNAseq analysis. The poly-(A) mRNA was enriched from the total RNA using oligo-(dT) magnetic beads. Following the enrichment, the mRNA was fragmented into small pieces in fragmentation buffer. These fragments served as templates for the first-strand cDNA synthesis using Superscript ${ }^{\mathrm{TM}}$ III reverse transcriptase and random hexamer (N6) primers. The RNA templates were removed, after which the second cDNA strand was synthesized using dNTPs, DNA polymerase $\mathrm{I}$, and RNase $\mathrm{H}$. The resulting short cDNA fragments were purified with AMPure XP beads. After the end-repair and A-tailing steps, the short cDNA fragments were ligated with the Illumina paired-end adapters and purified with AMPure XP beads. Next, a PCR was used to selectively enrich DNA fragments with adapters at both ends and prepare the final cDNA library, according to the kit's protocols. The concentrations of the cDNA libraries were determined using the Qubit 2.0 fluorometer (Life Technologies, Carlsbad, CA, USA) and their quality was evaluated using the Agilent 2100 Bioanalyzer. Finally, the 12 constructed libraries were sequenced from both ends using the Illumina $\mathrm{HiSeq}^{\text {тм }} 2500$ system (Illumina, San Diego,
CA, USA) at Beijing Novogene Science Co., Ltd. (Beijing, China).

\section{SMRT sequencing data processing and error correction}

SMRT raw sequencing data were filtered (parameters: minLength $=200$ and $\operatorname{minReadScore}=0.65$ ) using the SMRTlink 5.1 software to produce subreads. The CCS reads were generated from subread BAM files using the following parameters: min_length 50, max_drop_fraction 0.8, no_polish TRUE, min_zscore - 9999.0, min_passes 2, min_predicted_accuracy 0.8 , and max_length 15,000 . The CCS reads were then classified as Flnc reads and non-full length (NFL) reads using pbclassify.py, ignorepolyA false, and minSeqLength 200. The Flnc reads were then used for the isoform-level clustering (ICE) analysis. The resulting consensus sequence of clusters (CSC) was polished in a quiver program with NFL non-chimeric reads, using the following parameters: hq_quiver_min_ accuracy 0.99, bin_by_primer false, bin_size_kb 1, qv_ trim_5p 100, and qv_trim_3p 30 .

Additional nucleotide errors in the polished Flnc consensus sequence were corrected based on the Illumina RNA-seq data with the LoRDEC software. Redundant isoforms in the corrected consensus reads were removed with CD-HIT (-c 0.95 - T 6 -G 0 - aL $0.00-a S$ 0.99) to obtain the final non-redundant reference transcripts for the subsequent analyses.

\section{Gene functional annotation}

Non-redundant Flnc transcripts were annotated based on BLAST searches of the following seven databases: $\mathrm{Nr}$ (NCBI non-redundant protein sequences), $\mathrm{Nt}$ (NCBI non-redundant nucleotide sequences), Pfam (Protein family), KOG/COG (Clusters of Orthologous Groups of proteins), Swiss-Prot (a manually annotated and reviewed protein sequence database), KEGG (Kyoto Encyclopedia of Genes and Genomes), and GO (Gene Ontology). The programs used for the functional annotation included hmmscan (version: 3.1b2) for the Pfam database analysis, blast+ (version: $2.6 .0+$ ) for the $\mathrm{Nt}$ database analysis, and diamond blastx (version: 0.8.36) for the Nr, KOG/COG, Swiss-Prot, KEGG, and GO database analyses. The E-cutoff value for all seven database analyses was set as $\leq 1 \mathrm{e}-5$. The top hit for the BLAST results was used for the functional annotation. The open reading frame of each FL transcript was predicted using the ANGLE pipeline.

Hormone metabolism and signaling genes were annotated based on AraCyc (v17.1) and the KEGG database as well as the relevant published literature regarding Arabidopsis. Transcription factors and regulators were identified and classified using the iTAK program (version 1.7a) (https://github.com/kentnf/iTAK) and the associated database. 


\section{Gene expression levels and differential expression analysis}

The Illumina RNA-seq clean reads for 12 samples were separately mapped onto the above-mentioned Flnc transcript sequences using the bowtie 2 program in the RSEM software. The mapped read count of each transcript was calculated and further transformed into FPKM values, which were used as the expression levels in different samples. The DEGs between two compared stages were determined using the DESeq $R$ package (1.10.1), with the following criteria: $\mid \log _{2}$ (fold-change) $\mid \geq$ 1 and adjusted $p$ value $<0.05$.

The GO and KEGG enrichment analyses of the DEGs were performed using the GOseq $\mathrm{R}$ package (version 1.10.1) and the KOBAS software (version 2.0), respectively. The significance of the enriched GO terms and enriched KEGG pathways was separately assessed with the Wallenius non-central hyper-geometric distribution test and the hyper-geometric distribution test. An adjusted $p$ value $<0.05$ was set as the threshold for significance.

\section{qRT-PCR validation of differentially expressed genes}

To verify the transcriptome sequencing results, the expression levels of 15 unigenes involved in ABA/GA metabolism and signaling pathways in $P$. sibiricum seeds collected at the Seed, Corm, Str_S, and Eme stages were analyzed by qRT-PCR. Total RNA was extracted using the EASYspin Plus Complex Plant RNA kit (Aidlab, Beijing, China) and reverse transcribed to generate firststrand cDNA using the TUREscript 1st Strand cDNA Synthesis kit (Aidlab). The cDNA was used for the qRTPCR analysis involving gene-specific primers (Table S12) and $2 \times$ SYBR Green qPCR Master Mix (Aidlab). The qRT-PCR was performed using the CFX96 ${ }^{\text {rm }}$ Realtime PCR System (Bio-Rad, USA), with the following program: $94{ }^{\circ} \mathrm{C}$ for $3 \mathrm{~min} ; 40$ cycles of $94{ }^{\circ} \mathrm{C}$ for $15 \mathrm{~s}, 55^{\circ} \mathrm{C}$ for $15 \mathrm{~s}$, and $72{ }^{\circ} \mathrm{C}$ for $20 \mathrm{~s}$; and a melting curve analysis from 65 to $95^{\circ} \mathrm{C}$ for $5 \mathrm{~s}$. The $2^{-\Delta \Delta \mathrm{Ct}}$ method was used to calculate the expression levels of the analyzed genes [50]. A GAPDH gene that showed the most stablest expression among our four seed samples was used as the internal reference for normalizing and comparing the expression levels of the analyzed genes among different samples.

\footnotetext{
Abbreviations

P. sibiricum : Polygonatum sibiricum; MPD: Morphophysiological dormancy; SMRT: Single-molecule real-time; DEGs: Differentially Expressed Genes; qRTPCR: Quantitative real-time polymerase chain reaction; TF: Transcription factor; Iso-seq: Isoform sequencing; PacBio: Pacific Biosciences; CCS: Circular consensus sequence; Flnc: Full-length non-chimeric; FPKM: Fragments per kilobase of exon model per million mapped reads; ZEP: Zeaxanthin epoxidase; AAO3: Abscisic-aldehyde oxidase; CYP707A: Abscisic acid 8'hydroxylase; ABI5: Abscisic acid insensitive protein 5; ABF: ABA-responsive element-binding factor; PYL4/8: Abscisic acid receptor; PP2C: Protein phosphatase 2C; GA3ox: Gibberellin 3-oxidase; GAMT2: Gibberellin A4
}

carboxyl methyltransferase 2; GID1: Gibberellin-insensitive dwarf 1; GASA3/6/ 14: Gibberellin-regulation protein; CIGR2: Chitin-inducible gibberellinresponsive protein 2

\section{Supplementary Information}

The online version contains supplementary material available at https://doi. org/10.1186/s12870-021-03147-7.

\begin{abstract}
Additional file 1: The online version contains supplementary material available at XXX. Figure S1. Length distribution of the predicted coding sequences in $P$. sibiricum transcriptome. Figure S2. KOG annotation of $P$. sibiricum unigenes. Figure $\mathbf{S 3}$. GO annotation of $P$. sibiricum unigenes. Figure S4. FPKM distribution of $P$. sibiricum unigenes in different samples. Figure S5. PCA $(\mathbf{A})$ and Pearson correlation analysis $(\mathbf{B})$ of $P$. sibiricum samples. Figure S6. Expression patterns of differentiallyexpressed hormone metabolic and signaling genes during seed dormancy breaking and seedling establishment. Figure S9. The pipeline of studying molecular regulation of $P$. sibiricum seed dormancy and germination based on PacBio SMRT-Seq and Illumination RNA-Seq. Table S1. P. sibiricum unigenes containing different number of transcripts. Table S2. Statistics of functional annotation of P. sibiricum transcriptome. Table S7. RNA-seq mapped reads. Table S10. Total number/DEG number of each TF and TR family.
\end{abstract}

Additional file 2: Table S3. Homologous species distribution of $P$. sibiricum unigenes annotated in the $\mathrm{Nr}$ database.

Additional file 3: Table S4. P. sibiricum unigenes aligned to Polynatum genes in $\mathrm{Nr}$ database.

Additional file 4: Table S5. GO annotation of $P$. sibiricum unigenes. Additional file 5: Table S6. KEGG pathway annotation of $P$. sibiricum unigenes.

Additional file 6: Table S8. List of hormone metabolic and signaling genes in $P$. sibiricum transcriptome.

Additional file 7: Figure S7. qRT-PCR and RNA-seq results of representative DEGs involved in ABA metabolism and signaling pathway.

Additional file 8: Figure S8. $q R T-P C R$ and RNA-seq results of representative DEGs involved in GA metabolism and signaling pathway.

Additional file 9: Table S9. Transcription factors and regulators in $P$. sibiricum transcriptome.

Additional file 10: Table S11. List of P. sibiricum genes homologous to Arabidopsis known seed dormancy and germination genes.

Additional file 11: Table S12. List of $P$. sibiricum genes for RT-qPCR validation.

\section{Acknowledgments}

We thank Liwen Bianji, Edanz Editing China (www.liwenbianji.cn/ac), for editing the English text of a draft of this manuscript.

\section{Authors' contributions}

$D L$ and RA contributed equally to this study. DL analyzed RNA-seq data, wrote up and finalized the manuscript; RA performed partial RNA-seq data analysis and RT-qPCR and drafted the manuscript; JW, funding acquisition; DW: supervision; XL: study conception; JQ: funding acquisition, supervision, experimental design, data curation, manuscript drafting and finalization. All authors have read and approved the manuscript.

\section{Authors' information}

DL, dqliao@implad.ac.cn; RA, ruipengan94@gmail.com; JW, jhwei@implad.ac. cn; XL, xianenli@yeah.net; JQ, jjqi@implad.ac.cn;

Received: date; Accepted: date; Published: date

\section{Funding}

This research was funded by the CAMS Initiative for Innovative Medicine (2016-I2M-2-003) and National Natural Science foundation of China (31471575). The funders had no role in tin the design of the study and collection, analysis, and interpretation of data and in writing the manuscript. 


\section{Availability of data and materials}

The raw sequence data have been deposited in the Genome Sequence Archive of the BIG Data Center (Beijing Institute of Genomics, Chinese Academy of Sciences, https://ngdc.cncb.ac.cn/) (Accession No. CRA003484). All data generated or analyzed during this study are available from the corresponding author on reasonable request.

\section{Declarations}

Ethics approval and consent to participate

Not applicable.

\section{Consent for publication}

Not applicable.

\section{Competing interests}

The authors declare that they have no competing interests.

\section{Author details}

${ }^{1}$ Institute of Medicinal Plant Development, Chinese Academy of Medical Sciences \& Peking Union Medical College, Beijing 100193, China. ${ }^{2}$ College of Life Science, Hebei Agricultural University, Baoding 071000, Hebei, China. ${ }^{3}$ The Key Laboratory of Plant Physiology and Molecular Pathology, Hebei province, Hebei Agricultural University, Baoding 071000, Hebei, China.

\section{Received: 7 December 2020 Accepted: 27 July 2021}

\section{Published online: 12 August 2021}

\section{References}

1. Zhao P, Zhao CC, Li X, Gao QZ, Huang LQ, Xiao PG, et al. The genus Polygonatum: a review of ethnopharmacology, phytochemistry and pharmacology. Ethnopharmacol. 2018;214:274-91. https://doi.org/10.1016/j. jep.2017.12.006

2. Liu XQ, Yi H, Yao L, Ma HW, Zhang JY, Wang ZM. Advances in plants of Polygonatum and discussion of its development prospects. Chin Pharm. 2017:52:530-4

3. Cui XW, Wang SY, Cao H, Guo H, Li YJ, Xu FX, et al. A review: the bioactivities and pharmacological applications of Polygonatum sibiricum polysaccharides. Molecules. 2018;23(5):1170. https://doi.org/10.3390/ molecules23051170.

4. Chinese Pharmacopoeia Commission. Pharmacopoeia of the People's Republic of China. Beijing: China Medical Science Press; 2015.

5. Liu N, Dong ZH, Zhu XS, Xu HY, Zhao ZX. Characterization and protective effect of Polygonatum sibiricum polysaccharide against cyclophosphamideinduced immunosuppression in Balb/c mice. Int J Biol Macromol. 2018; 107(Pt A):796-802. https://doi.org/10.1016/j.ijbiomac.2017.09.051.

6. Zhang HY, Hu WC, Ma GX, Zhu NL, Sun XB, Wu HF, et al. A new steroidal saponin from Polygonatum sibiricum. J Asian Nat Prod Res. 2018;20(6):58692. https://doi.org/10.1080/10286020.2017.1351436.

7. Jo K, Kim H, Choi HS, Lee SS, Bang MH, Suh HJ. Isolation of a sleeppromoting compound from Polygonatum sibiricum rhizome. Food Sci Biotechnol. 2018;27(6):1833-42. https://doi.org/10.1007/s10068-018-0431-0.

8. Ha E, Hong H, Kim TD, Hong G, Lee S, Kim S, et al. Efficacy of Polygonatum sibiricum on mild insomnia: a randomized placebo-controlled trial. Nutrients. 2019;11(8). https://doi.org/10.3390/nu11081719.

9. Liu J, Zhu X, Wang WX, Ye HD, Ye YR, Hai MR. Seeds dormancy of Polygonatum sibiricum red.: research progress. J Agric. 2018;8(3):11-5.

10. Wu WC, Luo HC. The biologic characteristic of seeds for Polygonatum sibiricum. J Chin Med Mater. 1995;18(12):597-8.

11. Zhang YJ, Li YG, Wang W, Fan S, Zhang XY. Studies on seed morphology and germinating characteristics of Polygonatum sibiricum Red. Seed. 2009; 28(12):28-30 34.

12. Fu FL, Ding ZM, Ma CD, Wei JH, Li XE, Qi JJ. Studies on germination and emergence characteristic of Polygonatum sibiricum red seed. Modern Chinese Med. 2017;19(8):1151-6.

13. Zhang XF, Liu XD, Liu HW. Reproductive biology of Lilium dahuricum (IV)dynamic morphological anatomy of germination of the seed. J Northeast Forestry Univ. 1994;22(5):50-3.

14. Dhyani A, Phartyal SS, Nautiyal BP, Nautiyal MC. Epicotyl morphophysiological dormancy in seeds of Lilium polyphyllum (Liliaceae). J Biosci. 2013;38(1):13-9. https://doi.org/10.1007/s12038-012-9284-5.
15. Pickett FL. The germination of seeds of Arisaema. Proc Indiana Acad Sci. 1913;23:125-8.

16. Yang J, Lovett-Doust J, Lovett-Doust L. Seed germination patterns in green dragon (Arisaema dracontium, Araceae). Am J Bot. 1999;86(8):1160-7. https://doi.org/10.2307/2656980.

17. Wang $J$, Chang H, Zhou ZL, Chen YY, Sun YY, Guo HB, et al. Developmental anatomy of Polygonatum sibiricum red during seed germination process. Acta Bot Boreal Occident Sin. 2013;33(8):1584-8.

18. Qi JJ, Zheng N, Zhang B, Sun P, Hu SN, Xu WJ, et al. Mining genes involved in the stratification of Paris polyphylla seeds using high-throughput embryo transcriptome sequencing. BMC Genomics. 2013;14(1):358. https://doi.org/1 0.1186/1471-2164-14-358.

19. Qi JJ, Sun P, Liao DQ, SunTY ZJ, Li XE. Transcriptomic analysis of American ginseng seeds during the dormancy release process by RNA-Seq. PLoS One. 2015;10(3):e0118558. https://doi.org/10.1371/journal.pone.0118558.

20. Ma LP, Yuan JH, He SL, Hu YH. Characterizations of three endogenous hormones during seed germination in Paeonia ostii. Henan Science. 2015;33(3):374-9.

21. Chen M, Lin JY, Jungim $H$, Julie MP, Russell $B$, Matteo $P$, et al. Seed genome hypomethylated regions are enriched in transcription factor genes. PNAS 2018;115(35):E8315-22. https://doi.org/10.1073/pnas.1811017115.

22. Wang $W Y, X u$ MY, Wang $Y$, Jamil M. Basal transcription factor 3 plays an important role in seed germination and seedling growth of rice. Biomed Res Int. 2014;54:465739-13. https://doi.org/10.1155/2014/465739.

23. Tao Z, Shen LS, Gu XF, Wang Y, Yu HZ, He Y. Embryonic epigenetic reprogramming by a pioneer transcription factor in plants. Nature. 2017; 551(7678):124-8. https://doi.org/10.1038/nature24300.

24. Huang Y, Feng CZ, Ye Q, Wu WH, Chen YF. Arabidopsis WRKY6 transcription factor acts as a positive regulator of abscisic acid signaling during seed germination and early seedling development. PLoS Genet. 2016;12(2): e1005833. https://doi.org/10.1371/journal.pgen.1005833.

25. Narsai R, Law SR, Carrie C, Xu L, Whelan J. In-depth temporal transcriptome profiling reveals a crucial developmental switch with roles for RNA processing and organelle metabolism that are essential for germination in Arabidopsis. Plant Physiol. 2011;157(3):1342-62. https://doi.org/10.1104/ pp.111.183129.

26. Jiang WB, Yu DQ. Arabidopsis WRKY2 transcription factor mediates seed germination and postgermination arrest of development by abscisic acid. BMC Plant Biol. 2009;9(1):96. https://doi.org/10.1186/1471-2229-9-96.

27. Wang JL, Chang H, Zhou ZL, Guo HB, Zhang YJ, Zhang X. Effect of differenttime ultrasonic wave treatment on germination of Polygonatum sibiricum red. Seed and seedling physiology. Seed. 2014;33(1):30-3.

28. Cheng QX, Cao D, Li YP, Liu F, Xi PZ, Li JC, et al. Optimization technology for breaking dormancy of Polygonatum sibiricum red. Seed. Acta Botan Boreali-Occiden Sin. 2016;25(12):1870-5.

29. Zhu WF, Wang JL, Chang H, Liu F, Zhang YJ, Zhang XY. A study on technology for breaking dormancy of Polygonatum sibiricum red. Seeds. Seed. 2013;32(4):13-5.

30. Zhang YJ, Zhang YC, Li YG, Liu F, Guo HB. Seed dormant characteristics of Polygonatum sibiricum red. Bull Botanical Res. 2010;30(6):753-7.

31. Baskin CC, Baskin JM. Seeds: ecology, biogeography, and evolution of dormancy and germination. 2nd ed. San Diego: Elsevier Inc; 2014. p. 133-5.

32. Wang Y, Bailey DC, Yin SK, Dong XH. Characterizing rhizome bud dormancy in Polygonatum kingianum: development of novel chill models and determination of dormancy release mechanisms by weighted correlation network analysis. PLoS One. 2020;15(4):e0231867. https://doi.org/10.1371/ journal.pone.0231867.

33. Wang Y, Liu XQ, Su H, Yin SK, Han CX, Hao DD, et al. The regulatory mechanism of chilling-induced dormancy transition from endo-dormancy to non-dormancy in Polygonatum kingianum Coll. Et Hemsl rhizome bud. Plant Mol Biol. 2019;99(3):205-17. https://doi.org/10.1007/s11103-018-0812-z.

34. Betts NS, Berkowitz O, Liu R, Collins HM, Skadhauge B, Dockter C, et al. Isolation of tissues and preservation of RNA from intact, germinated barley grain. Plant J. 2017;91(4):754-65. https://doi.org/10.1111/tpj.13600.

35. Bizouerne E, Buitink J, Vu BL, Vu JL, Esteban E, Pasha A, et al. Gene coexpression analysis of tomato seed maturation reveals tissue-specific regulatory networks and hubs associated with the acquisition of desiccation tolerance and seed vigour. BMC Plant Biol. 2021;21(1):124. https://doi.org/1 0.1186/s12870-021-02889-8.

36. Li Z, Tang J, Srivastava R, Bassham DC, Howell SH. The transcription factor bZIP60 links the unfolded protein response to the heat stress response in maize. Plant Cell. 2020;32(11):3559-75. https://doi.org/10.1105/tpc.20.00260. 
37. Zhang KL, Yao L, Zhang Y, Baskin JM, Baskin CC, Xiong Z, et al. A review of the seed biology of Paeonia species (Paeoniaceae): with particular reference to dormancy and germination. Planta. 2019;249(2):291-303. https://doi.org/1 0.1007/s00425-018-3017-4.

38. Cho JS, Jang BK, Lee SM, Lee IJ, Lee CH. Factors affecting the dormancy and germination of bleeding heart [Lamprocapnos spectabilis (L.) Fukuhara] seeds. Plant Biol (Stuttg). 2020;22(3):514-21. https://doi.org/10.1111/plb.13 089.

39. Jia ZC, Zhao BB, Liu S, Lu ZG, Chang B, Jiang HR, et al. Embryo transcriptome and miRNA analyses reveal the regulatory network of seed dormancy in Ginkgo biloba. Tree Physiol. 2020:tpaa023. https://doi.org/10.1 093/treephys/tpaa023.

40. Cheng JJ, Dabuxilatu LJ, Yang SB, Zhu X, Wang WX, Ye HD, et al. Physiological research on the after-ripening process of the Polygonatum cyrtonema Hua seed. Seed. 2018:37(10):31-5.

41. Penfield S. Seed dormancy and germination. Curr Biol. 2017;27(17):R874-8. https://doi.org/10.1016/j.cub.2017.05.050.

42. Shu K, Liu XD, Xie Q, He ZH. Two faces of one seed: hormonal regulation of dormancy and germination. Mol Plant. 2016;9(1):34-45. https://doi.org/10.1 016/j.molp.2015.08.010

43. Carrera-Castaño G, Calleja-Cabrera J, Pernas M, Gómez L, Oñate-Sánchez L. An updated overview on the regulation of seed germination. Plants (Basel). 2020;9(6):703. https://doi.org/10.3390/plants9060703.

44. Tuan PA, Kumar R, Rehal PK, Toora PK, Ayele BT. Molecular mechanisms underlying abscisic acid/gibberellin balance in the control of seed dormancy and germination in cereals. Front Plant Sci. 2018;9:668. https:// doi.org/10.3389/fpls.2018.00668.

45. Chahtane H, Kim W, Lopez-Molina L. Primary seed dormancy: a temporally multilayered riddle waiting to be unlocked. J Exp Bot. 2017;68(4):857-69. https://doi.org/10.1093/jxb/erw377.

46. Yang K, Yang L, Fan W, Long GQ, Xie SQ, Meng ZG, et al. Illumina-based transcriptomic analysis on recalcitrant seeds of Panax notoginseng for the dormancy release during the after-ripening process. Physiol Plant. 2019; 167(4):597-612. https://doi.org/10.1111/ppl.12904.

47. Gambl PE, Mullet JE. Inhibition of carotenoid accumulation and abscisic acid biosynthesis in fluridone-treated dark-grown barley. Eur J Biochem. 1986; 160(1):117-21. https://doi.org/10.1111/j.1432-1033.1986.tb09947.x.

48. Li AH, Jiang SY, Yang G, Li Y, Guo N, Chen T, et al. Molecular mechanism of seed dormancy release induced by fluridone compared with cod stratification in Notopterygium incisum. BMC Plant Biol. 2018;18(1):116 https://doi.org/10.1186/s12870-018-1333-2.

49. Wang HD, Cheng QX, Li YP, Wu YJ, Zhang XY, Liu F, et al. The relationship between the degrading enzyme of cell wall and the endosperm weakening of Polygonatum sibiricum red.Seeds. Seed. 2018;37(7):23-7.

50. Livak KJ, Schmittgen TD. Analysis of relative gene expression data using real-time quantitative PCR and the 2- $\Delta \Delta C T$ method. Methods. 2001;25(4): 402-8. https://doi.org/10.1006/meth.2001.1262.

\section{Publisher's Note}

Springer Nature remains neutral with regard to jurisdictional claims in published maps and institutional affiliations.

Ready to submit your research? Choose BMC and benefit from:

- fast, convenient online submission

- thorough peer review by experienced researchers in your field

- rapid publication on acceptance

- support for research data, including large and complex data types

- gold Open Access which fosters wider collaboration and increased citations

- maximum visibility for your research: over $100 \mathrm{M}$ website views per year

At $\mathrm{BMC}$, research is always in progress.

Learn more biomedcentral.com/submissions 